\title{
First normal stress difference and crystallization in a dense sheared granular fluid*
}

\author{
Meheboob Alam ${ }^{1 \dagger}$ and Stefan Luding ${ }^{(1,2)}$ \\ ${ }^{1}$ Institut für Computeranwendungen 1, Universität Stuttgart, \\ Pfaffenwaldring 27, 70569 Stuttgart, Germany \\ ${ }^{2}$ Particle Technology, DelftChemTech, TU-Delft, \\ Julianalaan 136, 2628 BL Delft, The Netherlands
}

(Dated: October 30, 2018)

\begin{abstract}
The first normal stress difference $\left(\mathcal{N}_{1}\right)$ and the microstructure in a dense sheared granular fluid of smooth inelastic hard-disks are probed using event-driven simulations. While the anisotropy in the second moment of fluctuation velocity, which is a Burnett-order effect, is known to be the progenitor of normal stress differences in $d i$ lute granular fluids, we show here that the collisional anisotropies are responsible for the normal stress behaviour in the dense limit. As in the elastic hard-sphere fluids, $\mathcal{N}_{1}$ remains positive (if the stress is defined in the compressive sense) for dilute and moderately dense flows, but becomes negative above a critical density, depending on the restitution coefficient. This sign-reversal of $\mathcal{N}_{1}$ occurs due to the microstructural reorganization of the particles, which can be correlated with a preferred value of the average collision angle $\theta_{a v}=\pi / 4 \pm \pi / 2$ in the direction opposing the shear. We also report on the shear-induced crystal-formation, signalling the onset of fluidsolid coexistence in dense granular fluids. Different approaches to take into account the normal stress differences are discussed in the framework of the relaxation-type rheological models.
\end{abstract}

\footnotetext{
* Physics of Fluids, vol. 15, no. 8 (2003)

$\dagger$ Present address: Engineering Mechanics Unit, JNCASR, Bangalore 560064, India; Email: meheboob@jncasr.ac.in
} 


\section{INTRODUCTION}

In the last decade, a lot of research activity took place to unveil the properties of granular materials $^{1,2}$, primarily because of their industrial importance, but also due to their fascinating properties. This has unraveled many interesting and so far unresolved phenomena (for example, clustering, size-segregation, avalanches, the coexistence of gas, liquid and solid, etc.). Under highly excited conditions, granular materials behave as a fluid, with prominent non-Newtonian properties, like the normal stress differences ${ }^{3}$. While the normal stress differences are of infinitesimal magnitudes in a simple fluid (e.g. air and water), they can be of the order of its isotropic pressure in a dilute granular gas ${ }^{4}$. From the modelling viewpoint, the presence of such large normal-stress differences readily calls for higher-order constitutive models ${ }^{5,6}$ even at the minimal level.

Studying the non-Newtonian behaviour is itself an important issue, since the normal stresses are known to be the progenitors of many interesting and unique flow-features (e.g. rod-climbing or Weissenberg-effect, die-swelling, secondary flows, etc. ${ }^{7}$ ) in non-Newtonian fluids. Also, normal stresses can support additional instability modes (for example, in polymeric fluids and suspensions ${ }^{7-10}$, which might, in turn, explain some flow-features of granular fluids. For example, particle-clustering ${ }^{11-13}$ has recently been explained from the instability-viewpoint using the standard Newtonian model for the stress tensor ${ }^{12,14,15}$

The kinetic theory of Jenkins \& Richman ${ }^{16}$ first showed that the anisotropy in the second moment of the fluctuation velocities, due to the inelasticity of particle collisions, is responsible for such normal stress behaviour. They predicted that the first normal stress difference (defined as $\mathcal{N}_{1}=\left(\Pi_{x x}-\Pi_{y y}\right) / p$, where $\Pi_{x x}$ and $\Pi_{y y}$ are the streamwise and the transverse components of the stress deviator, respectively, and $p$ is the isotropic pressure, see section IIB) is maximum in the dilute limit, decreases in magnitude with density, and eventually approaches zero in the dense limit. Goldhirsch \& Sela ${ }^{4}$ later showed that the normal stress differences appear only at the Burnett-order-description of the Chapman-Enskog expansion of the Boltzmann equation. Their work has clearly established that the origin of this effect (in the dilute limit) is universal in both atomic and granular fluids, with inelasticity playing the role of a magnifier and thus making it a sizeable effect in granular fluids. While the source of the normal stress differences in the dilute limit has been elucidated both theoretically and by simulation, its dense counterpart has not received similar attention so far. This is an important limit since the onset of dilatancy (volume expansion due to shear ${ }^{17,18}$ ), crystallization, etc. occur in the dense regime, which in turn would influence the normal stress differences.

Previous hard-sphere simulations ${ }^{19,3,11}$ did look at the normal stress differences, but they did not probe the dense limit in a systematic way. These simulations showed that the first normal stress difference vanishes in the dense limit, in line with the theoretical predictions of Jenkins \& Richman ${ }^{16}$. On the other hand, the soft-sphere simulations of Walton \& Braun ${ }^{20}$, with frictional particles, showed that this quantity can change sign in the same limit. Our work with smooth inelastic hard-disks unequivocally demonstrates that $\mathcal{N}_{1}$, indeed, changes its sign at some critical density in the dense regime, due to the sign-change of its collisional component at a critical density, which depends on the value of the coefficient of restitution (e). More importantly, we show that the origin of $\mathcal{N}_{1}$ in the dense limit is distinctly different from that in a dilute granular gas. At the microstructural-level, certain topological changes in the anisotropic structure of the collision-angle distribution with density are responsible for the observed sign-reversal of $\mathcal{N}_{1}$. 
We use the familiar smooth hard-disk model for an event-driven simulation ${ }^{21}$ of the uniform shear flow configuration, focussing mainly on the normal stress behaviour and the microstructure formation as functions of the density and inelasticity. The details of the simulation technique and the relevant macroscopic quantities are described in section II. The simulation results on the first normal stress difference, the radial distribution function, the collision angle distribution and the crystalline-structure are presented in section III. Possible modelling approaches to incorporate the normal stress differences are discussed in section [V] In section $\nabla$ we summarize our findings, with suggestions for possible future work.

\section{SIMULATION METHOD}

We consider a collection of smooth inelastic hard-disks in a square box of size $\tilde{L}$ under uniform shear flow - let $\tilde{x}$ and $\tilde{y}$ be the streamwise and transverse directions, respectively, with the origin of the coordinate-frame being positioned at the centre of the box. The snapshot of a typical simulation, with non-dimensional coordinates, is shown in Fig. 1(a). Note that the dimensional quantities are denoted by tildes, and the reference length, time and velocity scales for non-dimensionalization will be specified later in this section.

Let the diameter and the mass of the particle be $\tilde{\sigma}$ and $\tilde{m}$, respectively. The pre- and post-collisional particle velocities of particle 1 are denoted by $\tilde{\mathbf{c}}_{1}$ and $\tilde{\mathbf{c}}_{1}^{\prime}$, respectively. Hence, the velocity of particle 2 relative to 1 is $\tilde{\mathbf{c}}_{21}=\tilde{\mathbf{c}}_{2}-\tilde{\mathbf{c}}_{1}$. Let $\mathbf{k}_{21}=\mathbf{k}$ be the unit vector directed from the center of particle 2 to that of particle 1 at contact. The pre- and post-collisional velocities are related by the expression:

$$
\mathbf{k} \cdot \tilde{\mathbf{c}}_{21}^{\prime}=-e\left(\mathbf{k} \cdot \tilde{\mathbf{c}}_{21}\right),
$$

where $e$ is the coefficient of normal restitution, with $0 \leq e \leq 1$; note that we restrict ourselves to perfectly smooth particles. The expression for the collisional impulse is

$$
\tilde{\mathbf{I}}=\tilde{m}\left(\tilde{\mathbf{c}}_{1}^{\prime}-\tilde{\mathbf{c}}_{1}\right)=\frac{\tilde{m}}{2}(1+e)\left(\mathbf{k} \cdot \tilde{\mathbf{c}}_{21}\right) \mathbf{k}
$$

directed along $\mathbf{k}$.

\section{A. Model system and algorithm}

The system is periodic in $\tilde{x}$-direction, i.e. a particle crossing the left/right boundary re-enters the system through the opposite boundary at the same vertical position with unchanged velocities. To impose a uniform shear rate $(\tilde{\gamma}=\tilde{U} / \tilde{L})$ in the $\tilde{y}$-direction, the top and bottom image boxes, bounding the central box, are set in motion with velocities $\tilde{U} / 2$ and $-\tilde{U} / 2$, respectively, in the streamwise direction. This is the standard approach to attain the state of uniform shear flow (USF) by imparting momemtum transfer by shearing, originally introduced by Lees \& Edwards ${ }^{22}$. Overall, this system represents an extended doubly-periodic system where the periodicity in the transverse direction is in the local Lagrangian frame. In a typical simulation, the disks are initially placed randomly in the computational box, and the initial velocity field is composed of the uniform shear and a small Gaussian random part. An event-driven algorithm is then used to update the system in time, the details of which may be found in Alam \& Luding ${ }^{23,24}$. 
To ascertain whether the system has reached the statistical steady-state, the time evolution of the average fluctuation kinetic energy ('granular' energy, defined in the next section) is monitored, see Fig. 1(b). Due to the balance between the shear work and the collisional dissipation under homogeneous shear deformation, the granular energy attains a constant value at the steady state. Depending on the value of the coefficient of restitution and the number of particles, it takes about thousand collisions per particle to reach such a statistical steady-state - the lower the value of $e$, the more quickly the system reaches the steady state and vice versa. The simulation is then allowed to run for another 15000 collisions per particle to gather data to calculate the macroscopic quantities. A few longer runs (30000 collisions per particle) were also checked, with no significant change on the measured quantities. Another quantity which was simultaneously monitored, along with granular energy, is the linearity of the streamwise velocity profile across the Couette gap, and we found that the calculated shear rate (i.e. the slope of the velocity profile) fluctuated around the imposed shear rate by at most $1 \%$ at densities where crystallization is not evidenced.

\section{B. Macroscopic quantities}

With $\tilde{L}, \tilde{\gamma}^{-1}, \tilde{\gamma} \tilde{L}$, and $\tilde{m}$, used as the reference length, time, velocity, and mass, respectively, the relevant dimensionless quantities are:

$$
\sigma=\frac{\tilde{\sigma}}{\tilde{L}}, \quad(\mathbf{c}, \mathbf{u}, \mathbf{C})=\frac{1}{\tilde{\gamma} \tilde{L}}(\tilde{\mathbf{c}}, \tilde{\mathbf{u}}, \tilde{\mathbf{C}}), \quad \mathbf{P}=\frac{\tilde{\mathbf{P}}}{\tilde{\rho} \tilde{\sigma}^{2} \tilde{\gamma}^{2}}, \quad T=\frac{\tilde{T}}{\tilde{\sigma}^{2} \tilde{\gamma}^{2}},
$$

where $\mathbf{u}$ is the 'hydrodynamic' velocity, $\mathbf{C}=\mathbf{c}-\mathbf{u}$ the fluctuation (peculiar) velocity of particles, $\tilde{\rho}$ the material density of particles, $\mathbf{P}$ the stress tensor, and $T$ the granular energy.

The macroscopic stress, as measured in discrete particle simulations, is a byproduct of the particle-level mechanisms of momemtum transfer. As in the hard-core model of dense gases, the stress is the sum of its kinetic and collisional components. The former arises from the transport of momentum as the particles move through the system carrying their momentum, while the latter is due to the direct interparticle collisions. The homogeneity of the uniform shear flow allows us to calculate the stress by averaging it over the whole computational box ${ }^{3,23,24}$.

The stress, defined in the compressive sense, may be decomposed in the standard way:

$$
\mathbf{P}=\mathbf{P}^{k}+\mathbf{P}^{c}=p \mathbf{1}+\mathbf{\Pi},
$$

where $p$ is the pressure, $\boldsymbol{\Pi}$ the pressure deviator and $\mathbf{1}$ the unit tensor. From the off-diagonal components of the pressure deviator, we can calculate the shear viscosity which relates the rate of strain to the shear stress:

$$
\mu=-\Pi_{x y} / \frac{\mathrm{d} u}{\mathrm{~d} y}
$$

For the steady uniform shear flow, thus, the dimensionless shear viscosity can also be interpreted as the shear stress due to our adopted scaling, $\mathrm{d} u / \mathrm{d} y=\gamma=1$. The diagonal components of the pressure deviator can be non-zero, giving rise to normal stress differences. The first normal stress difference is defined as

$$
\mathcal{N}_{1}=\frac{\left(\Pi_{x x}-\Pi_{y y}\right)}{p} .
$$


Note that we have scaled this quantity by pressure to ascertain its relative magnitude with respect to pressure. For a standard Newtonian fluid, $\mathcal{N}_{1}=0$ and thus $\mathcal{N}_{1}$ is an indicator of the non-Newtonian character of the fluid. $\mathcal{N}_{1}$ can be decomposed into kinetic and collisional parts:

$$
\mathcal{N}_{1}=\mathcal{N}_{1}^{k}+\mathcal{N}_{1}^{c}=\frac{\left(\Pi_{x x}^{k}-\Pi_{y y}^{k}\right)}{p}+\frac{\left(\Pi_{x x}^{c}-\Pi_{y y}^{c}\right)}{p} .
$$

Note that the sign of $\mathcal{N}_{1}$ crucially depends on the convention used to define the stress tensor. For example, in the rheology literature, stress is typically defined in the tensile sense $^{7}$. A positive $\mathcal{N}_{1}$ for the compressive case is equivalent to its negative value for the tensile case and vice versa. This point should be kept in mind while making any comparison with data in the rheology literature.

From the trace of the kinetic stress tensor, $\mathbf{P}^{k}$, one can calculate the granular energy,

$$
T=\frac{1}{2 \sigma^{2}}\left[\frac{1}{N} \sum_{i=1}^{N} C_{i} C_{i}\right]
$$

which is a measure of the random motion of the particles with respect to the mean motion.

There are two dimensionless control parameters: the volume fraction of particles $(\nu)$ and the coefficient of normal restitution $(e)$. The shear rate is also a control parameter, however, due to normalization we have $\gamma=\tilde{\gamma} / \tilde{\gamma}=1$, and changing the value of $\tilde{\gamma}$ does not influence the reported results; the imposed shear rate $\gamma$ is thus kept fixed at unity. The simulations are carried out for the whole range of solid volume fractions, varying from the dilute to the dense limit, over a large range of values for the coefficient of restitution $(e=0.3-0.99)$. For most of the simulations, the number of particles are fixed to $N=1024$, and increasing the value of $N$ by fourfold $(N=4096)$ did not affect the reported quantities noticeably; for example, the change in $\mathcal{N}_{1}$ was about $4.3 \%$ and $5.1 \%$ at $\nu=0.3$ and 0.75 , respectively, for a restitution coefficient of $e=0.9$. We note here that the system-size dependence of the rheological quantities (pressure and viscosity) is known to be strong only for a small number of particles $(N<100)^{11,24,25}$.

For the typical simulation in Fig. 1(a), at steady-state, after $2 \times 10^{7}$ collisions, the parameter values were $\nu=0.5, N=1024$ and $e=0.7$. The variations of the granular energy $T$ and the calculated shear rate $\gamma_{c a l}$ with time are shown in Fig. 1(b), along with corresponding initial variations in two insets. (The data represent the instantaneous values of $T$ and $\gamma_{c a l}$ sampled at a regular interval of 400 collisions - no time averaging is involved here.) Note that $\gamma_{c a l}$ was computed by binning the system into 20 equal-size bins in the transverse direction and taking averages over all particles in each bin. It is observed that the granular energy reaches its steady value $(T=0.6121 \pm 0.022)$ quickly after the initial transients and the calculated shear rate fluctuates around its imposed value $(\gamma=1)$ by about $\pm 1 \%$. The fluctuations in both $T$ and $\gamma_{c a l}$ at steady-state are due to the finite-size of the system and diminish with increasing number of particles as $N^{-1 / 2}$.

\section{RESULTS}

For detailed results on the quantities pressure, shear viscosity and granular energy, and for their comparison with kinetic theory predictions, we refer to our recent study ${ }^{24}$. Here, we will mainly focus on the behaviour of the first normal stress difference and its kinetic 
and collisional components. We also present results on the pair distribution function and the collision angle distribution to characterize microstructures. Lastly we will present results on crystal-formation at high densities, signalling the coexistence of fluid and solid, complementing recent results in non-sheared systems ${ }^{26,27}$.

\section{A. Normal stress difference}

Figure 2(a) shows the variation of the first normal stress difference $\left(\mathcal{N}_{1}\right)$ with density for two values of the coefficient of restitution. It is observed that $\mathcal{N}_{1}$ is maximum at the dilute limit and decreases thereafter with $\nu$. The overall variation of $\mathcal{N}_{1}$ with density looks similar at other values of the coefficient of restitution, with a difference in the magnitude of $\mathcal{N}_{1}$. The inset in Fig. 2(a) shows that $\mathcal{N}_{1}$ decreases quite sharply in the dense limit and becomes negative at some density $(\nu=\bar{\nu})$. Increasing the value of the restitution coefficient decreases this critical density $\bar{\nu}$. The arrows on the left-ordinate indicate the asymptotic values of $\mathcal{N}_{1}$ for a two-dimensional granular gas in the dilute limit ${ }^{4}$ :

$$
\mathcal{N}_{1}=1.0448\left(1-e^{2}\right) \text {. }
$$

The anisotropy in the second moment of the fluctuation velocity is primarily responsible for the finite normal stress difference in the dilute limit ${ }^{16,28}$ and this shows up only at the Burnett-order of the Chapman-Enskog expansion ${ }^{4}$. We should mention here that the limit $e \rightarrow 1$ is singular and the normal stress difference survives even in the elastic limit as pointed out by Goldhirsch \& Sela ${ }^{4}$. The corresponding expression for $\mathcal{N}_{1}$ in a molecular gas is:

$$
\mathcal{N}_{1} \approx 1.358 \frac{\gamma^{2} \ell^{2}}{\left\langle u^{2}\right\rangle}
$$

where $\ell$ is the mean free path and $\left\langle u^{2}\right\rangle$ is the rms of the velocity fluctuations. However, because of its extremely small magnitude under normal conditions, the normal stress difference is not measurable in a molecular fluid.

Previous hard-sphere simulations of Campbell and coworkers ${ }^{3,19}$ are in variance with our result in that they found $\mathcal{N}_{1} \rightarrow 0$ as $\nu \rightarrow \nu_{\max }$. However, the soft-sphere simulations of Walton \& Braun ${ }^{20}$, with frictional particles, support our observation that $\mathcal{N}_{1}$ indeed undergoes a sign-reversal. To better understand what is responsible for the sign-reversal of $\mathcal{N}_{1}$, we look at the kinetic and collisional components of the first normal stress difference. Figure 2(b) shows the variations of $\mathcal{N}_{1}^{k}$ and $\mathcal{N}_{1}^{c}$ with density at $e=0.7$. We observe that

$\mathcal{N}_{1}^{k}$ is maximum at the dilute limit and decreases monotonically to zero as $\nu$ approaches the packing limit. Except for the dense limit, the overall behaviour of $\mathcal{N}_{1}^{k}$ represents that of the total normal stress difference. The collisional component, $\mathcal{N}_{1}^{c}$, shows a non-monotonic variation with density: $\mathcal{N}_{1}^{c}$ is zero in the dilute limit, increases with increasing $\nu$, remains almost constant for intermediate densities, and then decays sharply in the dense limit. Interestingly, $\mathcal{N}_{1}^{c}$ becomes negative at some critical density $(\nu=\bar{\nu})$ beyond which the behaviour of $\mathcal{N}_{1}^{c}$ mirrors that of $\mathcal{N}_{1}$ (see inset). Thus the normal stress behaviour in the dense regime is clearly due to the anisotropy in the collisional stress.

Recall that the kinetic theory of Jenkins \& Richman ${ }^{16,28}$ predicts that $\mathcal{N}_{1} \rightarrow 0$ in the dense limit. The predictions of the revised Enskog theory of Santos et al. ${ }^{29}$ are in line with that of Jenkins \& Richman, even though their kinetic model is claimed to be valid even in the crystalline-phase. Since the source of normal stress differences in all these theories is 
linked to the anisotropy in the second moment of velocity fluctuations (which vanishes as $\left.\nu \rightarrow \nu_{\max }\right)$, they are unable to predict the correct behaviour of normal stresses in the dense limit. We would like to stress here that, as mentioned in the Introduction, many fascinating non-Newtonian effects ${ }^{7}$ are primarily determined by the first normal stress difference and its sign. For example, the rod-climbing effect can occur in a granular fluid if $\mathcal{N}_{1}<0$, i.e. only in the dense limit (assuming that the second normal stress difference is negligible). Furthermore, having a constitutive model which reproduces the correct sign of $\mathcal{N}_{1}$ is also important, since it is well known in the rheology literature that the odd sign for $\mathcal{N}_{1}$ leads to the instability of the rest state $^{30}$.

The sign-reversal of $\mathcal{N}_{1}^{c}$ can be succinctly presented as a phase-diagram in the $(\nu, e)$-plane by plotting the zeros of $\mathcal{N}_{1}^{c}$ as a function of the coefficient of restitution, see Fig. 3. Below the solid line, $\mathcal{N}_{1}^{c}$ is positive, and negative above it. Also plotted in this figure is the line for the zeros of $\mathcal{N}_{1}$ which, as expected, lies slightly above. Thus, the first normal stress difference is zero along the solid line which may be called the symmetry-line. It is observed that decreasing the coefficient of restitution increases the critical density $(\bar{\nu})$ at which $\mathcal{N}_{1}^{c}$ changes sign. As we approach the elastic limit, $\bar{\nu}$ depends strongly on the value of $e$. We further note that as $e \rightarrow 1, \bar{\nu} \sim 0.62$ which is well below the freezing-point density of a $2 \mathrm{D}$ hard-disk fluid, $\nu_{f} \approx 0.70^{26,27}$.

We need to mention here that the sign-change of $\mathcal{N}_{1}$ is not uncommon in other nonNewtonian fluids. For example, in non-Brownian viscous suspensions, $\mathcal{N}_{1}$ changes sign at high Peclet number ${ }^{31,32}$. However, the reason for this effect is quite different in granular fluids as we show below.

\section{B. Microstructural features}

To understand the microstructural mechanism for the origin of the first normal stress difference and its sign-reversal, here we probe several microstructural features of a dense granular fluid.

\section{Radial distribution function}

Typical snapshots of the system in the dense regime are shown in Fig. 4 at four different densities with $e=0.7$. Note that the density for the subplot $4(c)$ is $\nu=0.725$ for which $\mathcal{N}_{1}^{c} \approx 0$. Looking at the corresponding distribution of granular energies (not shown here for brevity), we could find signatures of clusters (group of particles) with lower energies surrounded by particles with higher energy. To understand the flow-microstructures and their energetics at such high densities, we need to probe the pair distribution function and similar measures for the structure of the packing.

Figure 5(a-d) shows the radial distribution function $g(r)$ at four densities, with parameter values as in Fig. 4. The thin, dotted lines are data from a non-sheared, homogeneous, elastic system ${ }^{26,27}$, whereas the thick, solid lines represent a sheared situation with rather strong dissipation $e=0.7$. (Note that the elastic distribution function has been measured under non-sheared periodic boundary conditions; also for $e$ somewhat smaller than unity, the same results were obtained as long as the system remains homogeneous.) We observe that the weak difference at low density $\nu=0.6$ grows with increasing density, concerning two aspects: 
(i) The peak value of contact in the sheared systems is always larger than that in a homogeneous system of the same density, and the difference increases strongly with density, another indicator for clustering ${ }^{33}$.

(ii) The peaks and valleys, which allow to distinguish between different lattice structures, are different in the sheared case when compared to the non-sheared situation. In the former case, peaks at $r / \sigma=1,2,3, \ldots$ are observed, signalling shell-formation about any test-particle. In the latter case, the peaks at $r / \sigma=1, \sqrt{3}, 2, \ldots$ indicate a crystallization transition and the development of a triangular lattice.

The peaks in the sheared situation, e.g. at $r / \sigma=2,3$, become sharper as the density is increased, but the one at $r / \sigma=4$ is not well-defined even at $\nu=0.75$; higher order peaks are almost invisible, indicating long-range disorder due to the shearing in contrast to the long-range order that evolves in the non-sheared system. The comparison between the sheared and non-sheared cases suggest that the structure-formation is much slower in a sheared fluid which, in turn, implies that, as expected, the freezing-point density of the former would be larger than that of the latter. The splitting of the second-peak in the nonsheared case corresponds to the onset of freezing transition ${ }^{34}$; with increasing density this splitting becomes much more prominent, with similar structural-features appearing at the successively higher-order peaks. For the sheared system, however, we do not observe similar splitting, rather we see a sharp second-peak. This also occurs for a highly inelastic sheared fluid at a much lower density ${ }^{33}$; hence, a sharp second peak is a signature of short-range ordering due to the dissipative particle-clustering. The higher-order peaks in the sheared case become prominent only if we go beyond $\nu=\pi / 4$, which corresponds to the limit of perfect square-packing, but it is difficult to maintain homogeneous-shearing at such high densities, because the system splits into two parts, a dense, cold, crystalline area and a dilute, hot, fluid area - see below for details.

Figure 6 shows the variations of the pressure and viscosity functions $\left(f_{p}=p / \rho T\right.$ and $f_{\mu}=\mu / \rho \sigma \sqrt{T}$, respectively) with density at a restitution coefficient $e=0.7$. We observe that both increase monotonically with density, much beyond $\nu=0.7001$, and we did not find the hysteretic van-der-Walls loop in our pressure data upto $\nu=\pi / 4$, another indication that the crystallization is hindered/delayed by shear. These observations, together with our result that in the elastic limit $\mathcal{N}_{1}$ changes sign at a much lower density $(\nu \approx 0.62$, see Fig. 3$)$ than the corresponding freezing-density, suggest that the sign-reversal of $\mathcal{N}_{1}$ is not related to the freezing-transition.

\section{Collision angle distribution}

Associated with the sign-reversal of the first normal stress difference is a change in the relative magnitudes of the normal stress components $\left(P_{x x}\right.$ and $\left.P_{y y}\right)$. Subtle changes in the direction and magnitudes of the collisional-mode of momemtum transfer could influence the individual components of the stress tensor. In order to test this hypothesis, we focus on the collision angle distribution function, $C(\theta)$, which is defined such that $C(\theta) d \theta$ is the probability of collisions occuring at an angle lying between $\theta$ and $\theta+d \theta$, with the angle $\theta$ being measured in the anticlockwise direction from the positive $x$-axis (see Fig. 13). For a fluid in equilibrium, all collisions are equally likely, and hence $C(\theta)$ is a uniform function of $\theta$, i.e. $C(\theta)=1 / \pi \approx 0.318309$. For a non-equilibrium system (e.g. shear flow), however, preferred collisions are dictated by the nature of the external field, leading to an anisotropic distribution for $C(\theta)^{35-38}$. Following Savage \& Jeffrey ${ }^{36}$ and Campbell \& Brennen ${ }^{37}$, an 
explicit expression for $C(\theta ; \nu, e)$ can be derived for the case of uniform shear flow as detailed in the Appendix. Note that the angular dependence of $C(\theta ; \nu, e)$ will be modified by both the density and the restitution coefficient.

Figure 7 shows the comparison of our simulation data on $C(\theta)$ with the theoretical predictions of equation (A9) for two different values of the restitution coefficient at a density $\nu=0.6$. It is observed that the probability of collisions is higher on the upstream-faces of the colliding particles, i.e. for $\theta \in[\pi / 2, \pi]$ and $\theta \in[-\pi / 2,0]$ (i.e. the hatched-areas on the test-particle in Fig. 13). This is a consequence of the imposed shear-field which compresses the flow-structure along the $3 \pi / 4$-direction and stretches it along the $\pi / 4$-direction. Regarding the comparison with theory, there is, clearly, a phase-difference between theory and simulation, and the overall agreement is only qualitative.

Note in Fig. 7 that the probability of collisions on the upstream-faces increases further as the restitution coefficient increases. This, in turn, suggests that particle motion becomes more streamlined (i.e. along the streamwise direction) with increasing dissipation levels, which will naturally lead to a reduction in the transverse component of the fluctuation velocities of the particles. Thus, the macroscopic manifestation of such microscopic streamlined-motion would be an increase in the magnitude of the kinetic component of the first normal stress difference $\left(\mathcal{N}_{1}^{k}\right)$.

Turning our attention to the range of densities where $\mathcal{N}_{1}$ undergoes a sign-reversal, we show the collision angle distributions $C(\theta)$ in Fig. 8 as polar plots with $e=0.7$; the corresponding densities are as in the subplots of Fig. 4. It is observed that the anisotropic structure of $C(\theta)$ gets further modified in this regime, with distinct peaks appearing near $\theta=$ 0 and $2 \pi / 3$ (see subplot $b$ ). While the peak at $\theta=0$ corresponds to head-on collisions between particles in the same-layer, the one at $\theta=2 \pi / 3$ clearly signals the onset of triangularstructure formation. Another noteworthy point is that the collisions on the downstream-faces of the colliding particles are rare at these densities and hence $C(\theta)$ can be approximated solely by its contributions from the second- and fourth-quadrants $(\theta \in[\pi / 2, \pi]$ and $\theta \in[-\pi / 2,0]$, respectively).

Since the momentum transfer occurs mainly due to collisions in the dense regime, the stress tensor can be approximated by

$$
\mathbf{P} \sim \int(\mathbf{k} \otimes \mathbf{k}) C(\theta) \mathrm{d} \theta
$$

where $\mathbf{k}$ is the unit vector joining the line of centres of the two colliding disks. Assuming now that all the collisions would occur at some average collision angle $\theta_{a v}$ so that $C(\theta)=$ $C\left(\theta_{a v}\right)$, and recalling that $C(\theta)$ is well represented in this regime by restricting $\theta$ only in the second- and fourth-quadrants, the expression for the first normal stress difference simplifies significantly to

$$
\mathcal{N}_{1} \sim\left[(\mathbf{k} \otimes \mathbf{k})_{x}-(\mathbf{k} \otimes \mathbf{k})_{y}\right]_{\theta=\theta_{a v}} C\left(\theta_{a v}\right) .
$$

It is trivial to check that $\mathcal{N}_{1}=0$ at $\theta_{a v}=-\pi / 4$. From our simulation data, we have calculated $\theta_{a v}$ by averaging $C(\theta)$ over the second- and fourth-quadrants, whose variation with density is plotted in Fig. 9 for two restitution coefficients. It is observed that $\theta_{a v}$ crosses through $-\pi / 4$ (i.e. $3 \pi / 4$ ) at around the critical density $\bar{\nu}$ for all restitution coefficients. For example, $\theta_{a v} \approx-45.16^{\circ}$ and $-45.04^{\circ}$ at $\bar{\nu}=0.725$ and 0.67 , respectively, where $\mathcal{N}_{1}$ changes sign. Thus, the microstructural signature of the sign-reversal of $\mathcal{N}_{1}$ is directly correlated with the average collision angle being greater or less than $-\pi / 4$ (or $3 \pi / 4$ ). 


\section{Crystallization: Fluid-solid coexistence}

Figure 10(a) shows a snapshot of the system at the steady state with parameter values being set to $\nu=0.8$ and $e=0.9$. It is observed that a solid-layer coexists with two fluidized zones on either side of it. A closer look into the solid-layer reveals that the particles are arranged in a triangular-packing, representing a crystal, and thus we have a clear evidence for fluid-solid coexistence. The corresponding instantaneous streamwise velocity profile at $t=390$ (i.e. the image-boxes have moved 390 strain units from their original position) is shown in Fig. 10(b); the coarse-graining is done by binning the system into 20 equal-size bins in the transverse direction and then taking averages over all the particles in each bin. Clearly, the shearing is inhomogeneous across the Couette-gap: the crystal is aligned along the streamwise direction and hence we call it a layered-crystal; the shear-rate in the fluidized regimes on either side of the crystal is almost uniform. Note that the asymmetric nature of the velocity profile also signals the breakdown of the Lees-Edwards boundary condition as a motor for the homogeneous shear. The formation and the time-evolution of this crystal can be ascertained from Fig. 10(c) which shows the corresponding evolution of the streamwise velocity at early times. We observe that the crystal has fully formed at $t=76$, and the velocity profile remains antisymmetric about $y=0$ till $t=150$. With further time-evolution, however, the crystal does not remain stationary in the transverse direction, rather it moves slowly with particles diffusing across the fluid-solid interface. The overall life-time of this crystal is several orders of magnitude larger than the external time-scale $\gamma^{-1}$, imposed by the shear. The corresponding collision-angle distribution $C(\theta)$ in Fig. $10(d)$ shows three distinct peaks at $\theta=0, \pi / 3$ and $2 \pi / 3$. Note that the peak at $\theta=\pi / 3$ does not exist in the fluid phase (refer to Figs. $8 a$ ), and this provides evidence that the particles in the crystalline-phase are arranged in the triangular-packing structure. The large area of the crystalline phase as well as its relatively large value of solids fraction (hence, small mean free time) further suggest that most of the collisions occurred in the crystalline-phase.

We should remark here that, at such high densities, the inelastic collapse $e^{39-41}$ would eventually terminate the evolution of the system. We used the TC-model ${ }^{40}$ to avoid inelastic collapse, but could not altogether eliminate it within the crystal after some time. But the important point to note is that the crystalline-phase can be maintained for a long period of time ( $t>100$ strain units), and hence the reported results are not transient effects.

Analogous plots for a nearly elastic system $(e=0.99)$ are shown in Fig. 11( $a-c)$ for the same density $\nu=0.8$. The overall features are similar to that for $e=0.9$, but the width of the crystalline zone is a little larger. By decreasing the dissipation-level to $e=0.7$, we did not observe crystal formation, with other parameters being fixed; by increasing the systemsize to $N=4096$, however, we observed layered crystal at $e=0.7$. Thus, the formation of such layered-crystalline structure depends crucially on the system size and the dissipation level: the larger the system-size and/or the weaker the dissipation, the more susceptible the system is to crystallize.

Note that even if we are well below the limit of perfect square-packing $\left(\nu_{s p}=\pi / 6 \approx\right.$ 0.785), the system could crystallize if the dissipation-levels are low; for example, we observed layered crystalline structures at $\nu=0.75$ with $e=0.99$ and $N=4096$. Decreasing the coefficient of restitution to $e=0.9$, the flow-field remained homogeneous. Thus, our layered crystalline structures appear to be tied to a long-wave instability of the elastic

hard-sphere fluids ${ }^{56}$. Since our results are not driven by the inelastic dissipation, they are distinctly different from the layered shear-banding patterns, as predicted by the kinetic the- 
ory models ${ }^{15,42,43}$, in granular Couette flows. Having said that we need to mention that such dissipative layering patterns (i.e. those which become stronger with increasing dissipation levels ${ }^{15}$ ) were also found in simulations of a dilute sheared granular fluid ${ }^{43}$, but the simulations were allowed to evolve from an unsheared initial configuration with a special kind of boundary condition. In contrast, all our simulations started from a uniform shear condition. In the present contribution, we have mainly focussed on the non-dissipative layering in the dense limit, and the related issues of dissipative layering are relegated to a future study.

Before moving further, we make a qualitative comparison with the earlier simulation work of Campbell \& Brennen ${ }^{37}$ on the bounded Couette flow of inelastic, frictional hard disks. They also reported similar layered-microstructure but due to the small system-size ( $N=40)$ and boundary-effects (they considered a shear-flow bounded by frictional walls), the distinct shear-band formation that we have reported is not evident in the snapshots of their simulations. Nevertheless, we believe that our results are akin to that reported by Campbell \& Brennen. One of the referees has drawn our attention to the recent work of Campbell ${ }^{44}$ who probed the dense limit of a three-dimensional Couette shear flow using softsphere simulations. He was able to maintain uniform shearing even at a density of $\nu=0.62$, but beyond that he reported shear-band formation in that the flow-field degenerates into sheared and non-sheared zones in the gradient direction. This simply suggests that one can maintain uniform shearing in a three-dimensional geometry even beyond the analogous square-packing limit $(\phi=\pi / 4 \approx 0.52)$ since the particles have now an additional degree of freedom, orthogonal to the shear plane, to rearrange themselves. Moreover, most of Campbell's simulations were done with 1000 particles (equivalently, 100 particles in 2D) at a restitution coefficient of 0.7 . With these parameter values, we did not find layered crystals at $\nu=0.8$ in two-dimensions (see discussion in the next section).

\section{Shear-induced ordering and Reynold's dilatancy}

One of the referees has drawn our attention to the recent work of Lutsko ${ }^{45}$ who studied the shear-induced ordering in a low-density $(\nu \approx 0.26)$ elastic hard-sphere fluid. The earliest simulations of Erpenbeck ${ }^{46}$ on 3D elastic hard-sphere fluids showed that at high shear rates the system breaks down into orderded (solid) and disordered (fluid) phases in the direction of the mean vorticity (i.e. normal to the shear-plane). This induces a long-range twodimensional ordering, called a string-phase, in the shear-plane. More importantly, such ordering occurs only for a range of shear-rates- the lower the density, the larger this shearrate interval. However, the latter work of Evans and Morriss ${ }^{47}$ showed that the disorder-order transition of Erpenbeck arises due to the profile-biased-thermostat ${ }^{47}$ since the string-phase vanishes completely when a profile-unbiased-thermostat ${ }^{47}$ is used. Thus, the shear-inducedordering in elastic hard-sphere fluids appears to depend on the choice of the thermostat. Note further that all the above works probed moderately dense systems only, well below the corresponding square-packing limit. Now to compare these results with our observations in granular fluids, we first need to define an equivalent shear rate since the granular energy and

the shear rate are dependent on each other for the latter system. From the energy balance equation, it is trivial to show that the granular energy has the following functional relation with the shear rate and the restitution coefficient:

$$
T \propto \frac{\gamma^{2}}{1-e^{2}} \propto \gamma^{* 2}
$$


where $\gamma^{*}=\gamma /\left(1-e^{2}\right)^{1 / 2}$ is defined as the reduced shear rate. In the quasielastic limit $(e \rightarrow 1)$ the reduced shear rate (and hence the granular energy) approaches infinity, which is equivalent to the high shear-rate limit of an elastic hard-sphere fluid. Since we observed layered crystalline structures only in the quasielastic limit, we may thus conclude that such structures would also persist in elastic hard-disk fluids at large shear rates.

It is interesting to ask whether the layered crystalline-structures of Figs. 10 and 11 are, in any way, related to the concept of the Reynold's dilatancy ${ }^{17,18}$ which is explained schematically in Fig. 12. The top two sketches depict the classical constant-load $d^{48}$ shear-cell experiment (in two-dimensions) in which the material is subjected to a constant normal load. Fig. 12(a) corresponds to an ideal situation of maximum packing, with the particles being arranged in a triangular lattice $\left(\nu=\nu_{t p}=\pi / 2 \sqrt{3}\right)$. Clearly, in this situation the top and the bottom plates of the shear cell will simply slide over the material, without deforming it. However, if one of the plates is allowed to move in the vertical direction and thereby allowing the particles to rearrange themselves, e.g. as in Fig. 12(b), the material can be deformed even homogeneously (if $\nu \leq \nu_{s p}=\pi / 6$ ). This is the shear-coupled volume change, commonly known as the Reynold's dilatancy. Note, however, that our simulations mimic constant-volume experiments, since the volume of the computational box remains fixed (see Figs. $12 c$ and $12 d$ ); but, of course, now the pressure (i.e. normal load) can vary in response to particle motions inside the shear cell. For this case also, the uniform shearing is possible if and only if the overall density remains below the square-packing limit $\left(\nu<\nu_{s p}\right)$. However, for densities above this value $\left(\nu_{s p}<\nu<\nu_{t p}\right)$, the shearing can be started only if we allow the particles to rearrange themselves. This is possible if a part of the system becomes denser, allowing free volumes to the rest of the system which is nothing but Reynold's dilatancy too. Hence, we will end up with a crystalline-phase coexisting with a fluid phase, a typical example of which is shown schematically in Fig. 12(d). Thus the phenomenon of Reynold's dilatancy, for densities $\nu_{s p}<\nu<\nu_{t p}$, would make the ordering transition, as depicted in Figs. 10 and 11, more prominent, and this effect would be much stronger in two-dimensions.

\section{CONSEQUENCES FOR THE CONSTITUTIVE MODELING: RELAXATION MODELS}

Here we attempt to describe the normal-stress behaviour of a granular fluid using the standard relaxation-type models. Prior literature on the dense-gas kinetic theory, which forms the foundation of theoretical developments of granular fluids in the rapid-shear regime, in-

dicates that such a stress relaxation mechanism does also exist in granular fluids ${ }^{49-51}$. The relaxation-type models are routinely used to describe the non-Newtonian behaviour of viscoelastic/viscoplastic materials, and hence might be apt for granular fluids in the dense limit as well. The recent work of Zhang \& Rauenzahn ${ }^{52-53}$ clearly shows that such viscoelastic stress relaxation mechanism exists in dense granular flows. Following a rigorous statistical mechanical procedure, they derived an evolution equation for the collisional stress tensor which boils down to a frame-indifferent viscoelastic model, with the Jaumann derivative appearing directly without appealing to objectivity arguments.

Let us consider the viscoelastic relaxation approximation suggested recently by Jin \& Slemrod ${ }^{51}$ to regularize the Burnett order equations of Sela \& Goldhirsch ${ }^{5}$ for a low-density 
granular fluid. Their proposed equation for the pressure deviator, in our notation, is

$$
\begin{aligned}
\mathbf{\Pi}+\tau_{1}\left(\frac{D \boldsymbol{\Pi}}{D t}-\right. & \left.\mathbf{L}^{T} \cdot \boldsymbol{\Pi}-\boldsymbol{\Pi} \cdot \mathbf{L}+\frac{2}{d} \operatorname{tr}(\boldsymbol{\Pi} \cdot \mathbf{L}) \mathbf{1}\right) \\
& +\tau_{2}\left(\mathbf{S} \cdot \boldsymbol{\Pi}+\boldsymbol{\Pi} \cdot \mathbf{S}-\frac{2}{d} \operatorname{tr}(\boldsymbol{\Pi} \cdot \mathbf{S}) \mathbf{1}\right)=\boldsymbol{\Pi}^{e q}
\end{aligned}
$$

where

$$
\begin{aligned}
\boldsymbol{\Pi}^{e q} & =-2 \mu \mathbf{S}-(\lambda \nabla \cdot \mathbf{u}) \mathbf{1}+\boldsymbol{\Pi}_{2}+\boldsymbol{\Pi}_{3}, \\
\mathbf{S} & =\frac{1}{2}\left(\mathbf{L}+\mathbf{L}^{T}\right)-\frac{1}{d}(\nabla \cdot \mathbf{u}) \mathbf{1}, \\
\mathbf{L} & =(\nabla \mathbf{u})^{T}=\left(\frac{\partial u_{i}}{\partial x_{j}}\right) \\
\lambda & =\left(\zeta-\frac{2}{d} \mu\right) \\
\tau_{1} & =0.3211\left(\frac{\mu}{p}\right) \\
\tau_{2} & =0.58775\left(\frac{\mu}{p}\right) .
\end{aligned}
$$

Here $\tau_{1}$ and $\tau_{2}$ are relaxation times, $d$ the dimensionality of the system, $\mathbf{L}$ is the velocity gradient, $\mathbf{S}$ the deviatoric part of the rate of strain tensor, $\mu$ the shear viscosity, $\zeta$ the bulk viscosity and $\mathbf{1}$ the identity tensor; $\boldsymbol{\Pi}_{2}$ and $\boldsymbol{\Pi}_{3}$ are higher order terms, explicitly written down in Jin \& Slemrod ${ }^{51}$. Note that both relaxation times are proportional to the ratio of the shear viscosity and the pressure, and hence proportional to the mean free time. In the limits of $\tau_{1}, \tau_{2} \rightarrow 0$ and $\Pi_{2}, \Pi_{3} \rightarrow 0$, we recover the standard Newtonian model for the stress tensor.

Neglecting the higher-order terms, an expression for the first normal stress difference can be obtained for the steady uniform shear flow:

$$
\mathcal{N}_{1}=\left[\frac{4 \tau_{1}}{2\left(1+\tau_{1}^{2}\right)+\tau_{2}\left(1+\tau_{1}-\tau_{2}\right)}\right]\left(\frac{\mu}{p}\right)>0 .
$$

This quantity is always positive, as in our simulation results for dilute flows, if the two relaxation times are of the same order.

It is important to note that the above evolution equation does not satisfy the principle of material frame indifference (MFI) which states that the constitutive laws should be invariant under rigid-rotation ${ }^{7,8}$. The scalar field $\phi$, the vector field $\mathbf{v}$ and the tensor field $\boldsymbol{\Pi}$ are called frame-indifferent or objective if the following relations hold for all $t$ :

$$
\begin{aligned}
\phi^{\prime}\left(\mathbf{x}^{\prime}, t^{\prime}\right) & =\phi(\mathbf{x}, t), \\
\mathbf{v}^{\prime}\left(\mathbf{x}^{\prime}, t^{\prime}\right) & =\mathbf{Q}(t) \mathbf{v}(\mathbf{x}, t), \\
\mathbf{\Pi}^{\prime}\left(\mathbf{x}^{\prime}, t^{\prime}\right) & =\mathbf{Q}(t) \boldsymbol{\Pi}(\mathbf{x}, t) \mathbf{Q}(t)^{T},
\end{aligned}
$$

where $\phi, \mathbf{v} \& \boldsymbol{\Pi}$ and $\phi^{\prime}, \mathbf{v}^{\prime} \& \boldsymbol{\Pi}^{\prime}$ are defined in two different frames $\mathcal{F}$ and $\mathcal{F}^{\prime}$, respectively, and $\mathbf{Q}(t)$ is a proper orthogonal tensor. Here $\mathcal{F}^{\prime} \in \mathcal{E}(\mathcal{F})$, with $\mathcal{E}(\mathcal{F})$ denoting the set of all frames obtainable from a given frame $\mathcal{F}$ by observer transformations. That the stress-tensor in a granular gas is not a frame-independent quantity (as in the hard-sphere gas ${ }^{54,55}$ ) is wellknown. Since the kinetic component of the first normal stress difference remains positive 
at all densities, the kinetic stress tensor can be modelled using a non-objective equation as discussed above. For the collisional stress tensor, one can postulate a similar evolution equation as in Eqn. (11), but $\tau_{1}$ must be multiplied by a factor which must change sign at the critical density. However, this equation would remain frame-dependent even in the dense limit. Thus, if one has to recover the Boltzmann-limit of relaxation-type equations, a frame-indifferent approach does not appear to work.

A question now arises as to the possibility of modelling normal stress differences using the standard frame-indifferent relaxation type models. The simplest way is to use either the lower-convected or the upper-convected equations for the pressure deviator:

$$
\begin{aligned}
& \boldsymbol{\Pi}+\alpha(\nu, e) \tau\left(\frac{\mathrm{D} \boldsymbol{\Pi}}{\mathrm{D} t}+\left\{\mathbf{L}^{T} \cdot \boldsymbol{\Pi}+\boldsymbol{\Pi} \cdot \mathbf{L}-\frac{2}{d} \operatorname{tr}(\boldsymbol{\Pi} \cdot \mathbf{L}) \mathbf{1}\right\}\right)=-2 \mu \mathbf{S}-(\lambda \nabla \cdot \mathbf{u}) \mathbf{1} \\
& \boldsymbol{\Pi}+\alpha(\nu, e) \tau\left(\frac{\mathrm{D} \boldsymbol{\Pi}}{\mathrm{D} t}-\left\{\mathbf{L} \cdot \boldsymbol{\Pi}+\boldsymbol{\Pi} \cdot \mathbf{L}^{T}-\frac{2}{d} \operatorname{tr}(\boldsymbol{\Pi} \cdot \mathbf{L}) \mathbf{1}\right\}\right)=-2 \mu \mathbf{S}-(\lambda \nabla \cdot \mathbf{u}) \mathbf{1}
\end{aligned}
$$

respectively. Here $\alpha(\nu, e)$ is an empirical constant, dependent on both the density and restitution coefficient. For both cases, the first normal stress difference is

$$
\mathcal{N}_{1}=-\frac{2 \tau \alpha}{1+\tau^{2} \alpha^{2}}\left(\frac{\mu}{p}\right)
$$

Clearly, if $\alpha(\nu, e)$ is obtained from simulation, its sign-reversal would also correspond to the sign-reversal of $\mathcal{N}_{1}$. Comparing the Jin-Slemrod equation with its corresponding frameindifferent analog (lower-convected model), we conclude that the loss of frame-indifference shows up as a sign-change of the first normal stress difference. It would be interesting to investigate whether one could relax the Sela-Goldhirsch equations ${ }^{4}$ using a frame-indifferent approach without violating the entropy inequality ${ }^{51}$.

Similarly, one could postulate evolution equations using other objective derivatives. In this regard, the co-rotational Jeffrey's model seems to be the ideal choice:

$$
\boldsymbol{\Pi}+\tau_{1}(\nu, e) \frac{\mathcal{D} \boldsymbol{\Pi}}{\mathcal{D} t}=-2 \mu\left(\mathbf{S}+\frac{\lambda}{2 \mu}(\nabla \cdot \mathbf{u}) \mathbf{1}+\tau_{2}(\nu, e) \frac{\mathcal{D} \mathbf{S}}{\mathcal{D} t}\right)
$$

with $\mathcal{D} / \mathcal{D} t$ being the Jaumann derivative ${ }^{7}$. The corresponding first normal stress difference is

$$
\mathcal{N}_{1}=-\frac{2\left(\tau_{1}-\tau_{2}\right)}{1+\tau_{1}^{2}}\left(\frac{\mu}{p}\right)
$$

which is positive/negative depending on whether $\tau_{1}$ is less/greater than $\tau_{2}$. Thus, the frameindifferent relaxation models are able to predict positive and negative first normal stress differences. (For the steady homogeneous shear flow, one can also model positive/negative normal stress differences by postulating a general orthonormal basis, generated by the nilpotent basis tensors, which satisfies the objectivity requirement; for related issues, the reader is referred to Goddard $^{8}$.)

\section{SUMMARY AND CONCLUSION}

We have probed the non-Newtonian behaviour and the incipient crystalline-phase in a sheared, monodisperse, two-dimensional granular fluid. The standard event-driven technique is used to simulate a box of hard-disks under homogeneous shear deformation. The 
information about the stress tensor is obtained by decomposing it in the standard way: $\mathbf{P}=p \mathbf{1}+\boldsymbol{\Pi}$, where $p$ is the pressure and $\boldsymbol{\Pi}$ the pressure deviator. The non-Newtonian behaviour is quantified in terms of the first normal stress difference: $\mathcal{N}_{1}=\left(\Pi_{x x}-\Pi_{y y}\right) / p$.

The granular fluid is non-Newtonian with a measurable first normal stress difference $\left(\mathcal{N}_{1}\right)$ which is positive (if the stress is defined in the compressive sense) in the dilute limit. Interestingly, however, $\mathcal{N}_{1}$ changes from positive to negative at a critical density in the dense regime. By decomposing $\mathcal{N}_{1}$ into the kinetic and collisional contributions, $\mathcal{N}_{1}=\mathcal{N}_{1}^{k}+\mathcal{N}_{1}^{c}$, we found that while $\mathcal{N}_{1}^{k}$ is always positive and decays to zero in the dense limit, $\mathcal{N}_{1}^{c}$ has a non-monotonic variation with density. In particular, $\mathcal{N}_{1}^{c}$ increases from zero in the dilute limit as $\nu$ increases, reaches a maximum at some value of $\nu$ and then decreases, eventually becoming negative in the dense limit. The density at which $\mathcal{N}_{1}^{c}=0(\nu \equiv \bar{\nu})$ depends crucially on the level of micro-scale dissipation; in particular, $\bar{\nu}$ increases as the coefficient of restitution decreases. We have constructed a phase-diagram in the $(\nu, e)$-plane by identifying the regions where $\mathcal{N}_{1}$ is positive/negative.

We have shown that the origin of the first normal stress difference, in the dense limit, is tied to shear-induced collisional anisotropies. The underlying mechanism is distinctly different from that is known for a dilute granular gas ${ }^{4,16}$ where the anisotropy in the second moment of the fluctuation velcoity, which is a Burnett-order effect, gives rise to normal stress differences.

At the micro-level, the particles undergo reorganization as the dense-limit is approached. The signatures of microstructural-reorganization have been captured by probing the collision-angle distribution, $C(\theta)$, which is anisotropic due to the presence of the mean shear. In particular, we have found that the topology of the anisotropic-structure of $C(\theta)$ changes, with collisions occurring at certain preferred angles on the upstream-faces of the colliding pairs. The sign-reversal of $\mathcal{N}_{1}$ is correlated with a preferred value of the average collision angle, $\theta_{a v}=\pi / 4 \pm \pi / 2$, averaged over the upstream-faces of the colliding particles.

The time evolution of the sheared granular fluid leads to crystallization in the dense limit, signalling the coexistence of fluid and solid. The particles are arranged in a triangularpacking inside the crystal, and it moves as a layer in the streamwise direction. The formation of such layered-crystalline structure depends crucially on the system size and the dissipation level: the larger the system-size and the weaker the dissipation, the more susceptible the system is to crystallize. This appears to be related to a long-wave instability ${ }^{56}$ of the elastic hard-sphere fluids.

The present work clearly shows that the available kinetic-theory-based rheological models for granular fluids are not adequate to predict the behaviour of the first normal stress difference in the dense limit. Certain microstructural-features, like the preferred distribution of collisions which eventually leads to crystal-formation, should be incorporated into the theory. At such high densities, many-body effects (both positional and velocity correlations) are important ${ }^{57,41}$ and the appropriate kinetic description is the BBGKY-hierarchy ${ }^{57}$. To incorporate the observed normal stress behaviour into the framework of plausible constitutive models, we showed that the standard frame-indifferent relaxation type models can be used to model both positive and negative first normal stress differences. In this regard, the twoparameter Jeffrey's model ${ }^{7}$ appears to be the ideal choice; however, we are unable to recover the corresponding Boltzmann limit which is known to be non-objective. On the whole, we believe that a lot remains to be done for a better understanding of the dense-phase rheology of granular fluids even in the hard-sphere limit. 


\section{ACKNOWLEDGEMENT}

M.A. acknowledges the financial support by the Alexander von Humboldt Foundation and S.L. acknowledges the support of the Deutsche Forschungsgemeinschaft. M.A. is grateful to Hans J. Herrmann for discussions and hospitality at ICA1, and also acknowledges discussions with Joe D. Goddard on certain aspects of normal stress behaviour in granular fluids. We thank John F. Brady for directing us to relevant references on normal stresses in suspensions. We also thank three anonymous referees for their constructive comments.

\section{APPENDIX A: SHEAR-INDUCED ANISOTROPY AND THE COLLISION ANGLE DISTRIBUTION}

Here we are interested in the shear-induced anisotropy of the collision angle distribution of an inelastic hard-disk fluid. Following Savage \& Jeffrey ${ }^{36}$, an expression for the collision angle distribution $C(\theta)$ is derived, which is compared with the simulation data in IIB.2.

To calculate the probablility of collisions at a specific angle $\theta$, we focus on Fig. 13 with two particles colliding at $\mathbf{r}$. Note that $\theta$ is measured anticlockwise from the positive $x$-axis. For collisions to occur in a time $\delta t$, the center of particle 1 must lie inside the volume $\sigma \delta \mathbf{k}(\mathbf{q} \cdot \mathbf{k}) \delta t$, where $\mathbf{q}=\mathbf{c}_{1}-\mathbf{c}_{2}$ is the relative velocity of the colliding pair. Thus the expected total number of collisions (per unit time and unit area) with the line of centres $\mathbf{k}$ lying between $\mathbf{k}-\delta \mathbf{k} / 2$ and $\mathbf{k}+\delta \mathbf{k} / 2$ is given by

$$
\int \sigma f^{(2)}\left(\mathbf{c}_{1}, \mathbf{r}_{1}, \mathbf{c}_{2}, \mathbf{r}_{2}\right)(\mathbf{q} \cdot \mathbf{k})(\mathbf{k} \cdot \mathbf{n}) d \mathbf{k} d \mathbf{c}_{1} d \mathbf{c}_{2}
$$

where $f^{(2)}(\cdot)$ is the two-particle distribution function which is defined so that $f^{(2)}\left(\mathbf{c}_{1}, \mathbf{r}_{1}, \mathbf{c}_{2}, \mathbf{r}_{2}\right) d \mathbf{c}_{1} d \mathbf{c}_{2} d \mathbf{r}_{1} d \mathbf{r}_{2}$ is the number of pairs of particles such that the particle $i$ is located in an area element $d \mathbf{r}_{1}$ about $\mathbf{r}_{1}$ with its velocity in the interval $d \mathbf{c}_{1}$ about $\mathbf{c}_{1}$ while particle $j$ is located in an area element $d \mathbf{r}_{2}$ about $\mathbf{r}_{2}$ with its velocity in the interval $d \mathbf{c}_{2}$ about $\mathbf{c}_{2}$. To progress further, we have to invoke the assumption of molecular chaos and hence the expected number of collisions is

$$
\int \sigma g\left(\mathbf{r}_{1}, \mathbf{r}_{2}\right) f^{(1)}\left(\mathbf{c}_{1}, \mathbf{r}_{1} ; \mathbf{u}\left(\mathbf{r}_{1}\right)\right) f^{(1)}\left(\mathbf{c}_{2}, \mathbf{r}_{2} ; \mathbf{u}\left(\mathbf{r}_{2}\right)\right)(\mathbf{q} \cdot \mathbf{k})(\mathbf{k} \cdot \mathbf{n}) d \mathbf{k} d \mathbf{c}_{1} d \mathbf{c}_{2}
$$

where $g\left(\mathbf{r}_{1}, \mathbf{r}_{2}\right)$ is the pair-distribution function. For the steady uniform shear flow, $g\left(\mathbf{r}_{1}, \mathbf{r}_{2}\right)$ is calculated from the relation ${ }^{35,36,58}$ :

$$
g\left(\mathbf{r}_{1}, \mathbf{r}_{2}\right)=\frac{2 g_{c}(\nu)}{n^{2}} \int_{\mathbf{q} \cdot \mathbf{k}>0} f^{(1)}\left(\mathbf{c}_{1}, \mathbf{r}_{1} ; \mathbf{u}\left(\mathbf{r}_{1}\right)\right) f^{(1)}\left(\mathbf{c}_{2}, \mathbf{r}_{2} ; \mathbf{u}\left(\mathbf{r}_{2}\right)\right) d \mathbf{c}_{1} d \mathbf{c}_{2}
$$

where $g_{c}(\nu)$ is the contact value of the pair-distribution function and $\mathbf{q} \cdot \mathbf{k}>0$ implies that the integration be carried out for impending collisions.

As a first approximation, the single particle velocity distribution function $f^{(1)}\left(\mathbf{c}_{1}, \mathbf{r}_{1} ; \mathbf{u}\left(\mathbf{r}_{1}\right)\right)$ is assumed to have the Maxwellian-form:

$$
f^{(1)}\left(\mathbf{c}_{1}, \mathbf{r}_{1} ; \mathbf{u}\left(\mathbf{r}_{1}\right)\right)=\left(\frac{n m}{\pi k_{B} T}\right) \exp \left[-\frac{m\left(\mathbf{c}_{1}-\mathbf{u}\left(\mathbf{r}_{1}\right)\right)^{2}}{2 k_{B} T}\right],
$$


where $T$ is the granular temperature (fluctuating kinetic energy) and $k_{B}$ the usual Boltzmann constant. Now transforming the particle velocities $\left(\mathbf{c}_{1}, \mathbf{c}_{2}\right)$ to their center-of-mass and relative velocities, equation (A3) can be integrated to arrive at the following explicit expression for the pair-distribution function ${ }^{36}$ :

$$
g\left(\mathbf{r}_{1}, \mathbf{r}_{2}\right)=g_{c}(\nu) \operatorname{erfc}\left[\frac{2 \mathbf{k} \cdot \mathbf{u}\left(\mathbf{r}_{2}\right)}{\left(2 k_{B} T\right)^{1 / 2}}\right]
$$

where $\operatorname{erfc}(\cdot)$ is the complementary error function. Using the above expression for the pairdistribution function and transforming in terms of polar coordinates $(r, \theta)$, the integral for the normalized collision angle distribution yields ${ }^{37}$

$$
C(\theta)=\mathcal{A}(T)\left[\exp \left(-\frac{\sin ^{2} \theta \cos ^{2} \theta}{2 T}\right)-\frac{g(\theta) \sin \theta \cos \theta}{\sqrt{T}}\right] g(\theta),
$$

where $g(\theta)$ is the angular pair-distribution function given by

$$
g(\theta) \equiv \frac{g\left(\mathbf{r}_{1}, \mathbf{r}_{2}\right)}{g_{c}(\nu)}=\operatorname{erfc}\left[\frac{\sin \theta \cos \theta}{\sqrt{2 T}}\right]
$$

and $\mathcal{A}(T)$ is a normalization constant.

For the uniform shear flow, an expression for the granular temperature, can be obtained from the energy balance equation, by equating the energy production due to shear-work with the energy loss due to collisions:

$$
\begin{aligned}
\mu\left(\frac{d u}{d y}\right)^{2} & =\mathcal{D} \\
\Rightarrow T & =f_{\mu}(\nu) / f_{\mathcal{D}}(\nu, e)
\end{aligned}
$$

where $\mu=\rho_{p} \sigma f_{\mu}(\nu) \sqrt{T}$ is the shear viscosity and $\mathcal{D}=\left(\rho_{p} / \sigma\right) f_{\mathcal{D}}(\nu, e) T^{3 / 2}$ the collisional dissipation rate, with

$$
\begin{aligned}
f_{\mu}(\nu) & =\frac{\sqrt{\pi} \nu}{8}\left[\frac{1}{\nu g_{c}(\nu)}+2+\nu g_{c}(\nu)\left(1+\frac{8}{\pi}\right)\right], \\
f_{\mathcal{D}}(\nu, e) & =\frac{4}{\sqrt{\pi}}\left(1-e^{2}\right) \nu^{2} g_{c}(\nu) .
\end{aligned}
$$

Substituting this expression for $T$, the normalized collision distribution function becomes

$$
C(\theta ; \nu, e)=\mathcal{A}(T)\left[\exp \left(-\frac{f_{\mathcal{D}}(\nu, e) \sin ^{2} \theta \cos ^{2} \theta}{2 f_{\mu}(\nu)}\right)-g(\theta) \sin \theta \cos \theta \sqrt{\frac{f_{\mathcal{D}}(\nu, e)}{f_{\mu}(\nu)}}\right] g(\theta)
$$

and the angular pair-distribution function

$$
g(\theta ; \nu, e)=\operatorname{erfc}\left[\sin \theta \cos \theta \sqrt{\frac{f_{\mathcal{D}}(\nu, e)}{2 f_{\mu}(\nu)}}\right] .
$$


It is clear that the angular dependence of $C(\theta ; \nu, e)$ is modified by both the inelastic dissipation and the density.

[1] H.J. Herrmann, J.-P. Hovi and S. Luding, Physics of Dry Granular Media- NATO ASI Series E 350, (Kluwer Academic, Dordrecht, 1998).

[2] T. Pöschel and S. Luding, Granular Gases, Lecture Notes in Physics 764, (Springer, Berlin, 2001).

[3] C.S. Campbell, "Rapid granular flows," Ann. Rev. Fluid Mech., 22, 57 (1990).

[4] I. Goldhirsch and N. Sela, "Origin of normal stress differences in rapid granular flows," Phys. Rev. E., 54, 4458 (1996).

[5] N. Sela and I. Goldhirsch, "Hydrodynamic equations for rapid flows of smooth inelastic spheres to Burnett order," J. Fluid Mech., 361, 41 (1998).

[6] J.D. Goddard, "Dissipative materials as models of thixotropy and plasticity," J. Non-Newt. Fluid Mech., 14, 141 (1984).

[7] R.B. Bird, R.C. Armstrong and O. Hassager, Dynamics of Polymeric Liquids, (John Wiley Sons, London, 1977).

[8] J. D. Goddard, "Polymer fluid mechanics," Adv. Appl. Mech., 19, 143 (1977).

[9] J.D. Goddard and M. Alam, "Shear-flow and material instabilities in particulate suspensions and granular media," Particulate Science and Technology, 17, 69 (1999).

[10] P. Coussot, Q.D. Nguyen, H.T. Huynh and D. Bonn, "Avalanche behavior in yield stress fluids," Phys. Rev. Lett., 88, 175501 (2002).

[11] M.A. Hopkins and M.Y. Louge, "Inelastic microstructure in rapid granular flows of smooth disks," Phys. Fluids A, 3, 47 (1991).

[12] I. Goldhirsch and D. Zanetti, "Clustering instability in dissipative gases," Phys. Rev. Lett., 70, 1619 (1993).

[13] S. Luding and H.J. Herrmann, "Cluster growth in freely cooling granular media," Chaos, 9, 673 (1999).

[14] S. B. Savage, "Instability of unbounded uniform granular shear flow," J. Fluid Mech., 241, 109 (1992).

[15] M. Alam and P.R. Nott, "Stability of plane Couette flow of a granular material," J. Fluid Mech., 377, 99 (1998).

[16] J.T. Jenkins and R.W. Richman, "Plane simple shear of smooth inelastic circular disks: the anisotropy of the second moment in the dilute and dense limit,” J. Fluid Mech., 192, 313 (1988).

[17] J.D. Goddard and A.K. Didwania, "Computations of dilatancy and yield surfaces for assemblies of rigid frictional spheres," Quart. J. Mech. Appl. Math., 39, 145 (1997).

[18] M. Reiner, "A mathematical theory of dilatancy," Amer. J. Math., 67, 350 (1945).

[19] C.S. Campbell and A. Gong, "The stress tensor in a two-dimensional granular shear flow," J. Fluid Mech., 164, 107 (1986).

[20] O.R. Walton and R.L. Braun, "Viscosity, granular-temperature, and stress calculations for shearing assemblies of inelastic, frictional disks," J. Rheol., 30, 949 (1986).

[21] M.P. Allen and D.J. Tildesley, Computer Simulations of Liquids, (Clarendon Press, Oxford, 1989).

[22] A.W. Lees and S.F. Edwards, "The computer study of transport processes under extreme 
conditions," J. Phys. C, 5, 1921 (1972).

[23] M. Alam and S. Luding, "How good is the equipartition assumption for the transport properties of a granular mixture?" Granular Matter, 4, 139 (2002).

[24] M. Alam and S. Luding, "Rheology of bidisperse granular mixtures via event-driven simulations," J. Fluid Mech., 476, 69 (2003).

[25] E.D. Liss and B.J. Glasser, "The influence of clusters on the stress in a sheared granular material," Powder Tech., 116, 116 (2001).

[26] S. Luding, "Global equation of state of two-dimensional hard sphere systems," Phys. Rev. E., 63, 042201-1 (2001).

[27] S. Luding and O. Strauss, "The equation of state of polydisperse granular gases," in Granular Gases, Lecture Notes in Physics 564, T. Pöschel and S. Luding (eds.), Springer, 2001.

[28] M.W. Richman, "The source of second moment in dilute granular flows of highly inelastic spheres," J. Rheol., 33, 1293 (1989).

[29] A. Santos, J.M. Montanero, J.Dufty and J.J. Brey, "Kinetic model for the hard-sphere fluid and solid," Phys. Rev. E, 57, 1644 (1998).

[30] R.L. Fosdick and K.R. Rajagopal, "Anomalous features in the model of second order fluids," Arch. Rat. Mech. Anlys., 70, 145 (1979).

[31] J.F. Brady and M. Vivic, "Normal stresses in colloidal dispersions," J. Rheol., 39, 545 (1995).

[32] D.R. Foss and J.F. Brady, "Structure, diffusion and rheology of Brownian suspensions by Stokesian dynamics simulations," J. Fluid Mech., 407, 167 (2000).

[33] S. Luding, "Structures and non-equilibrium dynamics in granular media," Comptes Rendus Academie des Science, 3, 153 (2002).

[34] L. V. Woodcock and C. A. Angel, "Diffusivity of the hard-sphere model in the region of fluid metastability," Phys. Rev. Lett., 47, 1129 (1981).

[35] J.G. Kirkwood, F.P. Buff and M.S. Green, "The statistical mechanical theory of transport processes," J. Chem. Phys., 17, 988 (1949).

[36] S.B. Savage and D.J. Jeffrey, "The stress tensor in a granular flow at high shear rates," J. Fluid Mech., 110, 255 (1981).

[37] C.S. Campbell and C.E. Brennen, "Computer simulation of granular shear flows," J. Fluid Mech., 151, 167 (1985).

[38] J.T. Jenkins and E. Askari, "Boundary conditions for rapid granular flows: phase interfaces," J. Fluid Mech., 223, 497 (1991).

[39] S. McNamara and W.R. Young, "Inelastic collapse in two dimensions," Phys. Rev. E, 50, R28 (1994).

[40] S. Luding and S. McNamara, "How to handle the inelastic collapse of a dissipative hard particle gas with the TC model?" Granular Matter, 1, 113 (1998).

[41] S. Luding and A. Goldshtein, "Hydrodynamic theory for dissipative hard spheres with multiparticle interactions," Granular Matter (2003, in press).

[42] Nott, P.R., Alam, M., Agrawal, K., Jackson, R. and Sundaresan, S, "The effect of boundaries on the plane Couette flow of granular materials: A bifurcation analysis," J. Fluid Mech., 397, 203 (1999).

[43] M.-L. Tan, "Microstructures and macrostructures in rapid granular flows," Ph.D. thesis, Princeton (1995).

[44] C.S. Campbell "Granular shear flows at the elastic limit," J. Fluid Mech., 465, 261 (2002).

[45] J. F. Lutsko, "Molecular chaos, pair correlation, and shear-induced ordering of hard spheres," Phys. Rev. Lett., 77, 2225 (1996). 
[46] J. Erpenbeck, "Shear viscosity of the hard sphere fluid via nonequilibrium molecular dynamics," Phys. Rev. Lett., 52, 1333 (1984).

[47] D.J. Evans and G.P. Morriss, "Shear thickening and turbulence in simple fluids," Phys. Rev. Lett., 56, 2172 (1986).

[48] S.B. Savage and S. Sayed, "Stresses developed by dry cohesionless granular materials in an annular shear cell," J. Fluid Mech., 142, 391 (1984).

[49] H. Grad, "On the kinetic theory of rarefied gases," Comm. Pure and Appl. Math., 2, 331 (1949).

[50] J.T. Jenkins and M.W. Richman, "Grad's 13-moment system for a dense gas of inelastic spheres," Arch. Rat. Mech. Anal., 87, 355 (1985).

[51] S. Jin and M. Slemrod, "Regularization of the Burnett equations for rapid granular flows via relaxation," Physica D, 150, 207 (2001).

[52] D.Z. Zhang and R.M. Rauenzahn, "A viscoelastic model for dense granular flows," J. Rheol., 41, 1275 (1997).

[53] D.Z. Zhang and R.M. Rauenzahn, "Stress relaxation in dense and slow granular flows," J. Rheol., 44, 1019 (2000).

[54] E. Ikenberry and C. Truesdell, "On the pressures and flux of energy in a gas according to Maxwell's kinetic theory," J. Rat. Mech. Anal., 5, 1 (1956).

[55] I. Müller, "On the frame dependence of stress and heat flux," Arch. Rat. Mech. Anal., 45, 241 (1972).

[56] M. Lee, J. Dufty, J. Montanero, A. Santos and J. F. Lutsko, "Long wavelength instability for uniform shear flow," Phys. Rev. Lett., 76, 2702 (1996).

[57] R. Soto, J. Piasecki and M. Mareschal, "Precollisional velocity correlations in a hard-disk fluid with dissipative collisions," Phys. Rev. E, 64, 031306 (2001).

[58] S. Hess, "Structure of liquids and solids in nonequilibrium," J. de Physique, 46 (C3), 191 (1985). 

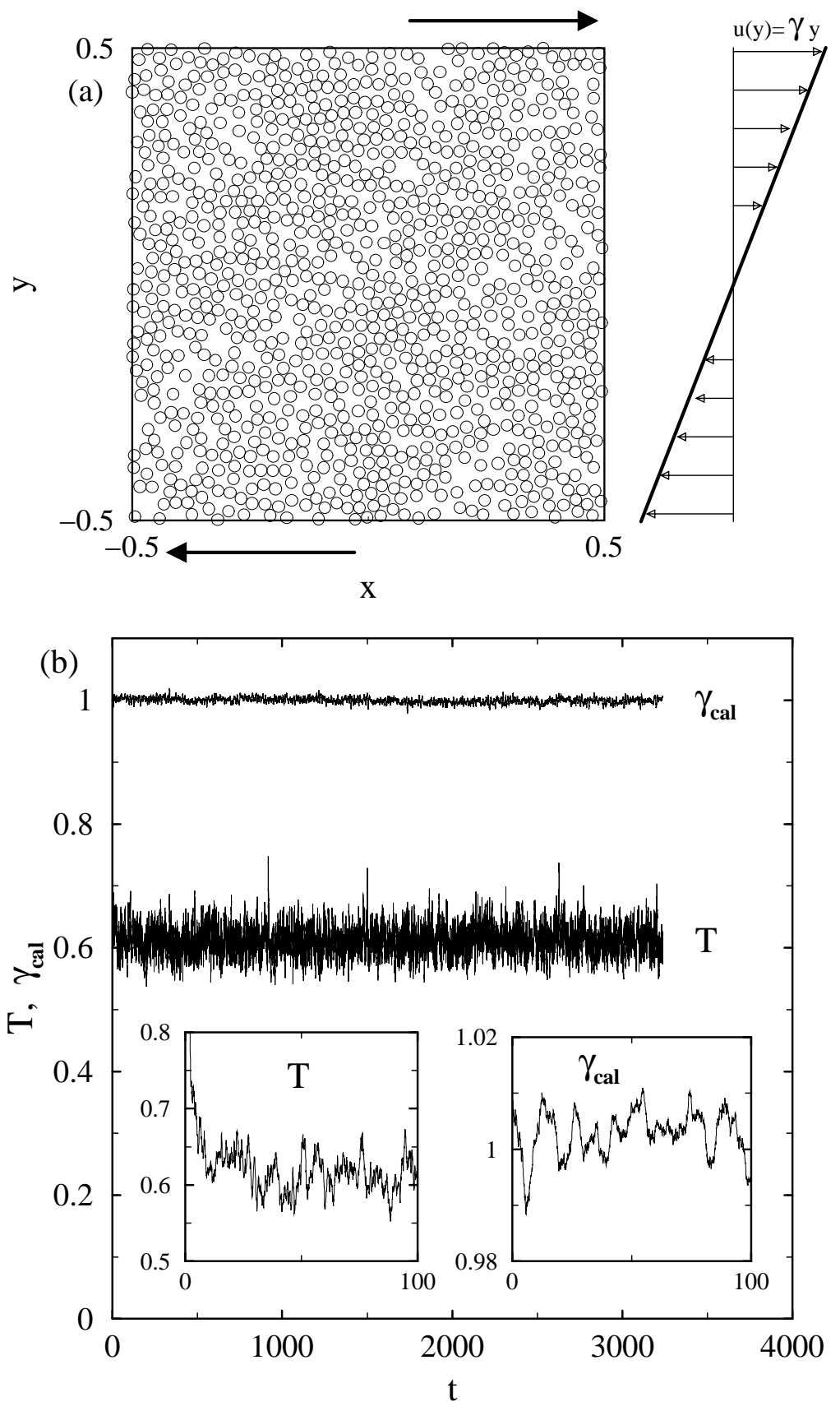

FIG. 1: (a) A snapshot of the sheared granular system at steady-state. The arrows indicate the displacement of the image boxes. (b) Variations of the granular energy $T$ and the calculated shear rate $\gamma_{c a l}$ with time. For an explanation of the system and particle properties, see the text. The parameters for both subplots are $\nu=0.5, e=0.7$ and $N=1024$. 

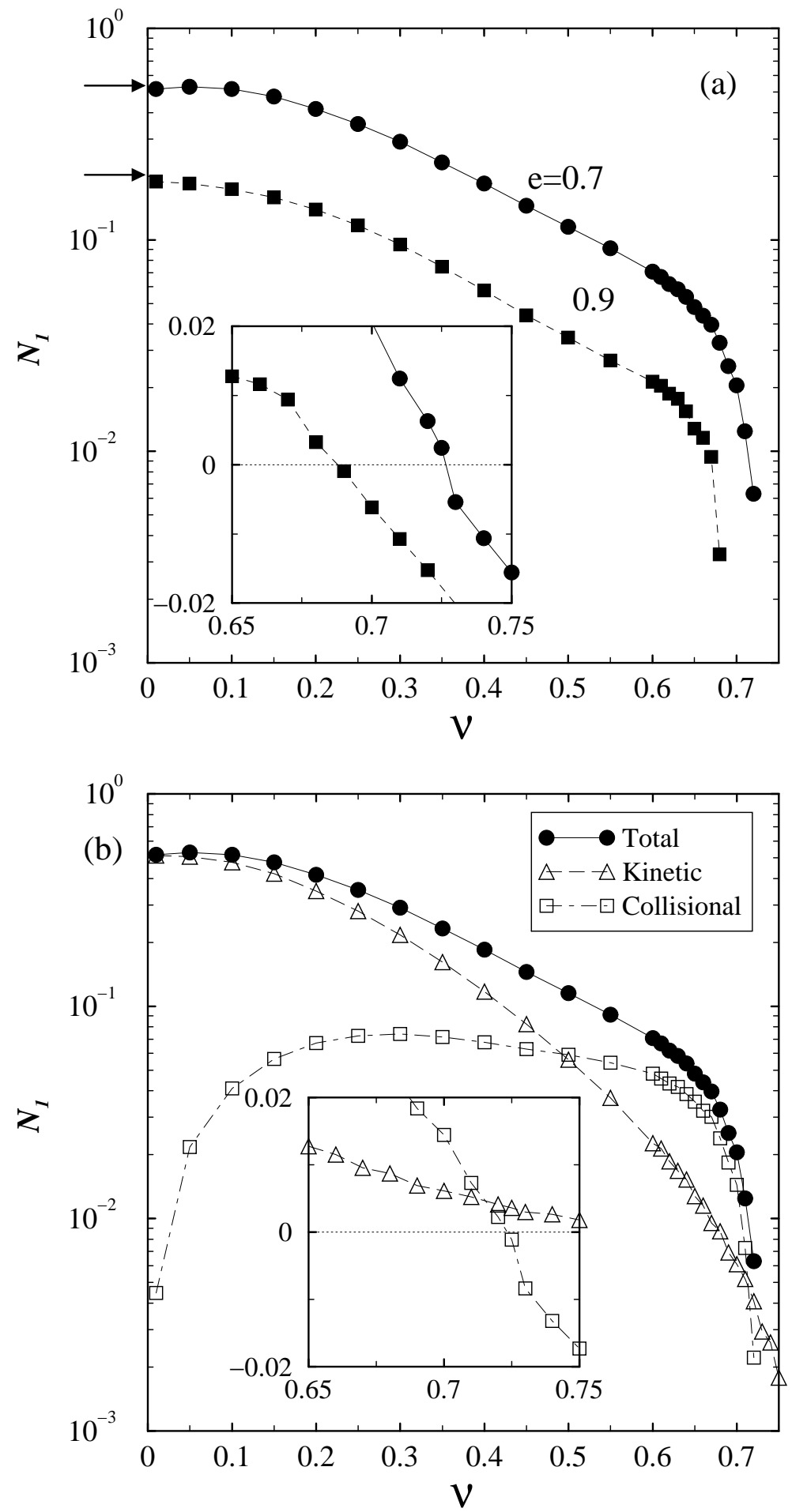

FIG. 2: (a) Variation of the first normal stress difference $\mathcal{N}_{1}$ with the solid volume fraction. The arrows on the left ordinate indicate corresponding analytical values for a two-dimensional granular gas. (b) Variations of $\mathcal{N}_{1}, \mathcal{N}_{1}^{k}$ and $\mathcal{N}_{1}^{c}$ with $\nu$ at $e=0.7$. In both subplots, the symbols represent the simulation data and the lines are drawn to guide the eye. 


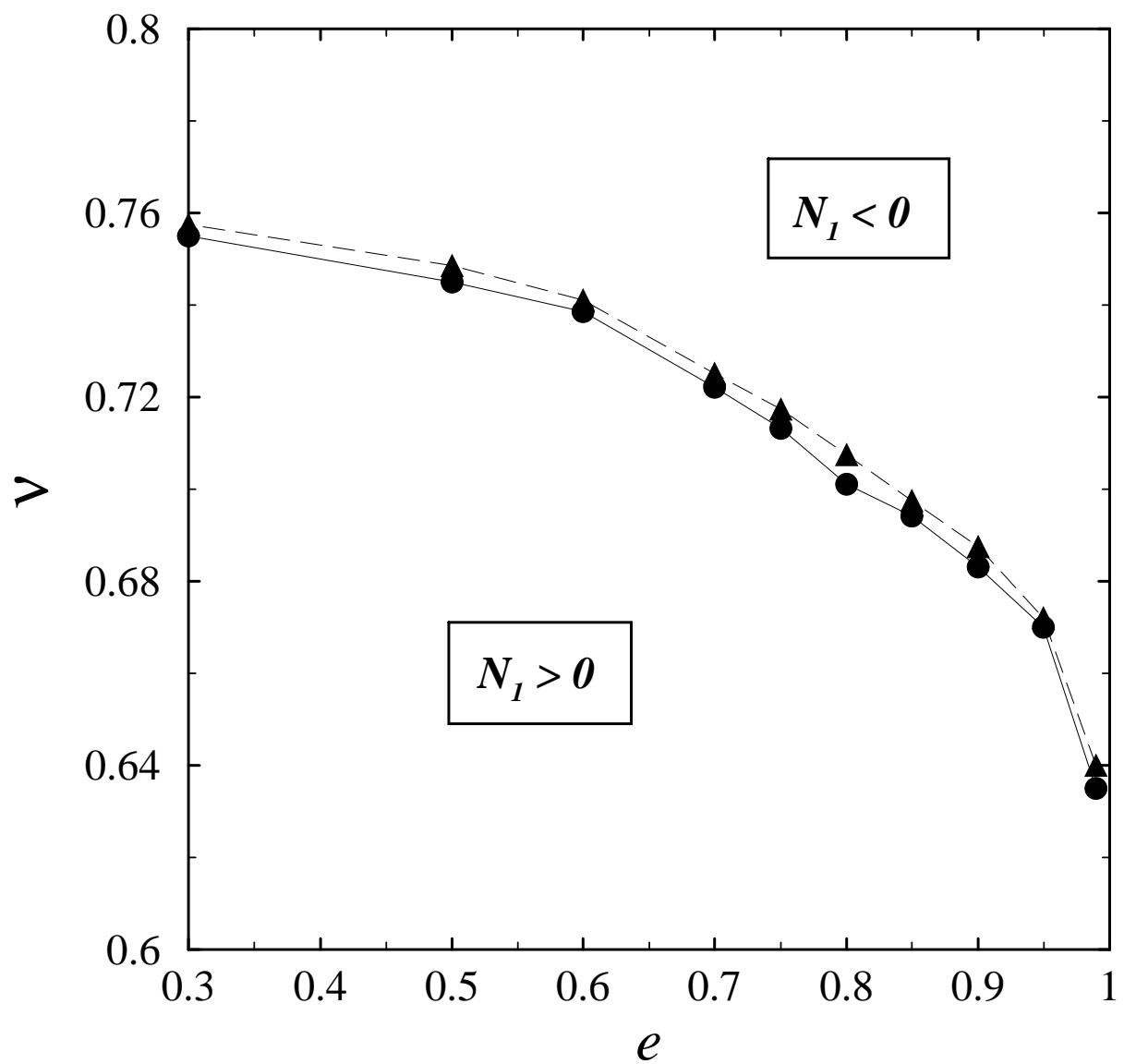

FIG. 3: The phase diagram, delineating regions of positive and negative first normal stress difference, in the $(\nu, e)$-plane. The filled circles and triangles represent zeros of $\mathcal{N}_{1}^{c}$ and $\mathcal{N}_{1}$, respectively. 

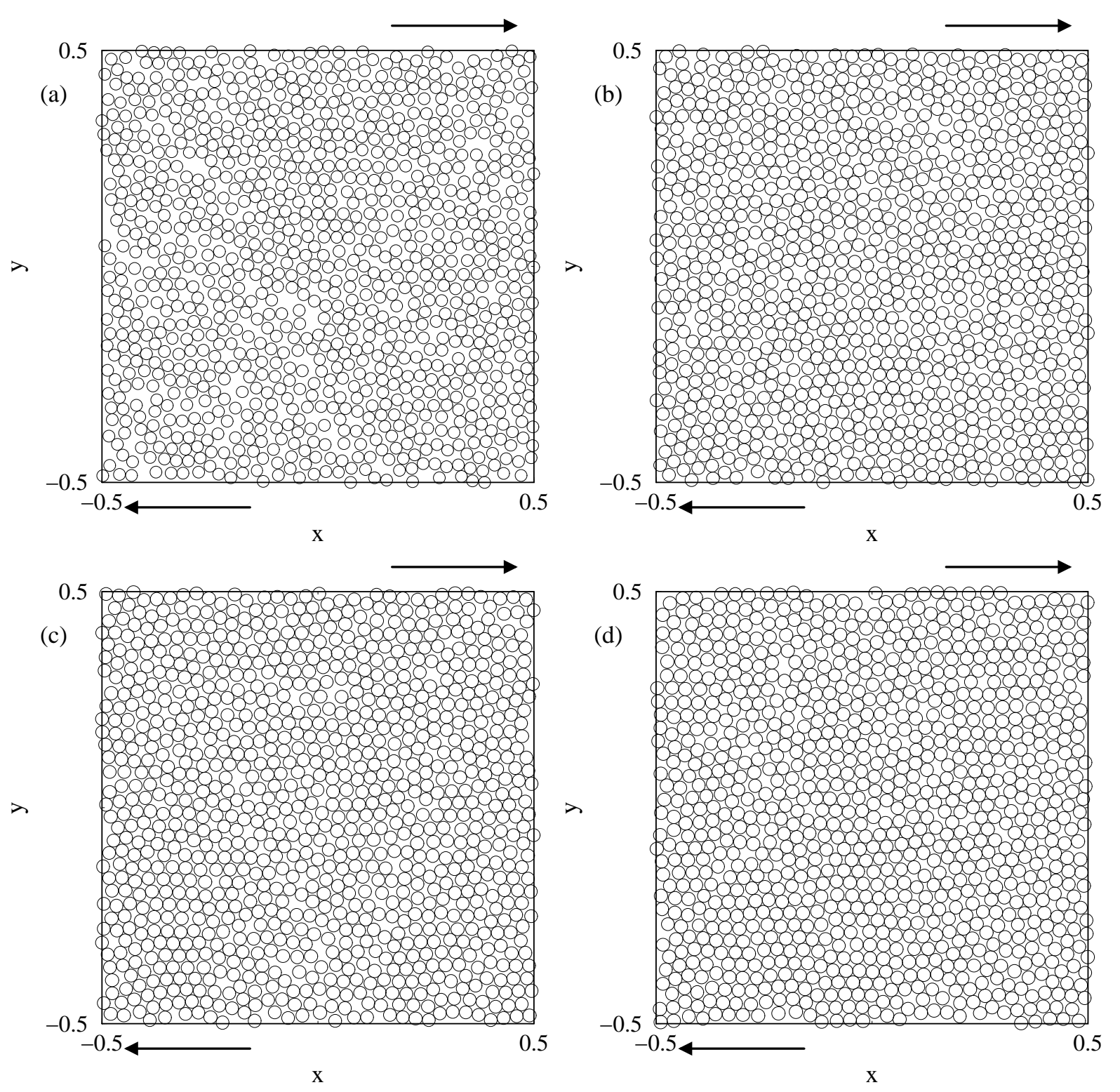

FIG. 4: Snapshots of the sheared system with $e=0.7$ in the dense limit for different densities: (a) $\nu=0.6$, (b) $\nu=0.7$; (c) $\nu=0.725 ;(d) \nu=0.75$. 

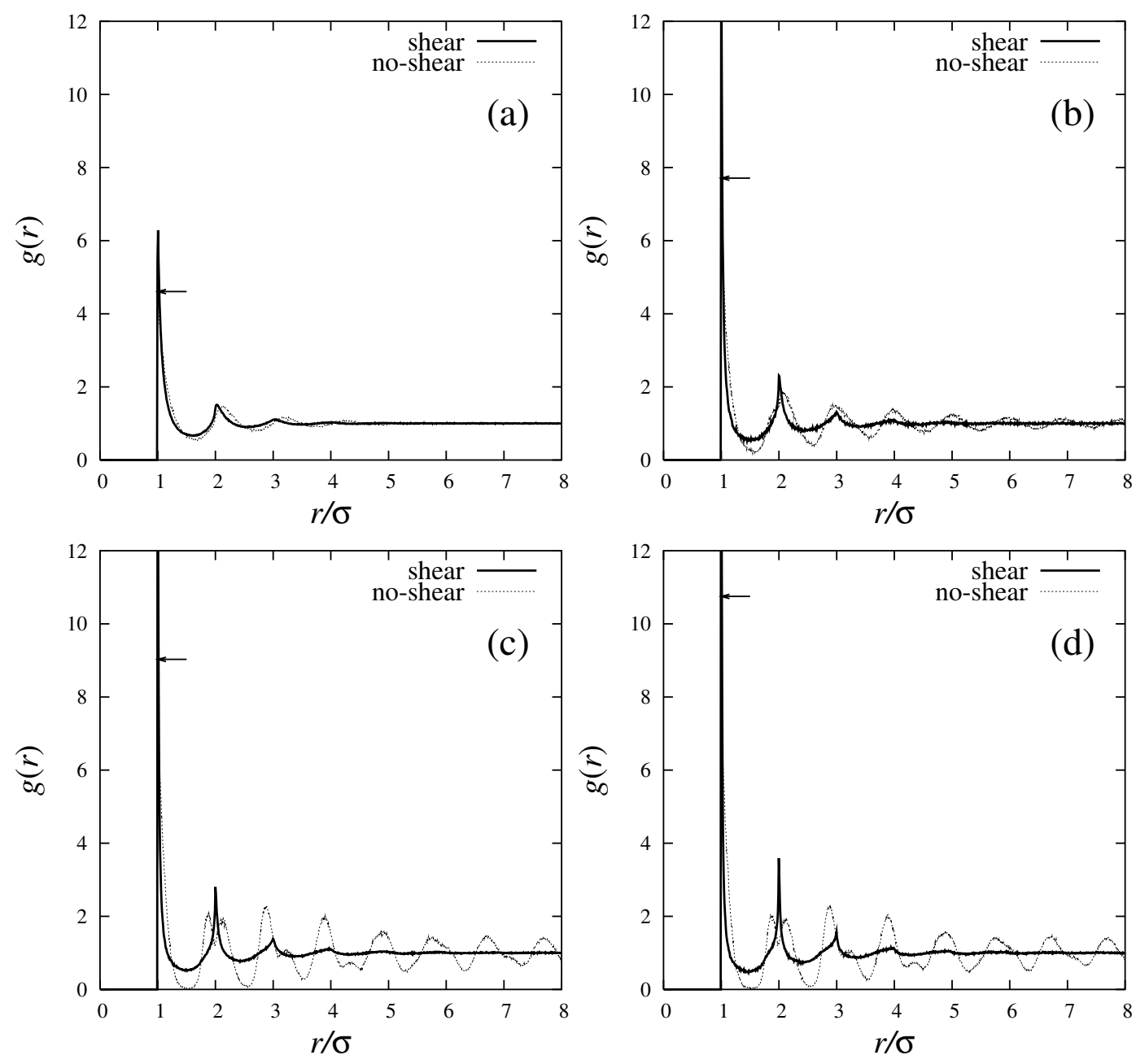

FIG. 5: Radial distribution function $g(r)$ plotted against the normalized distance $r / \sigma$ from sheared simulations with dissipation $e=0.7$ (solid lines) and from homogeneous, non-sheared situations with $\gamma=0$ and $e=1$ (dotted lines): (a) $\nu=0.6$, (b) $\nu=0.7 ;(c) \nu=0.725 ;(d) \nu=0.75$. The arrows indicate the peak values of $g(r)$ at contact for the homogeneous system. 


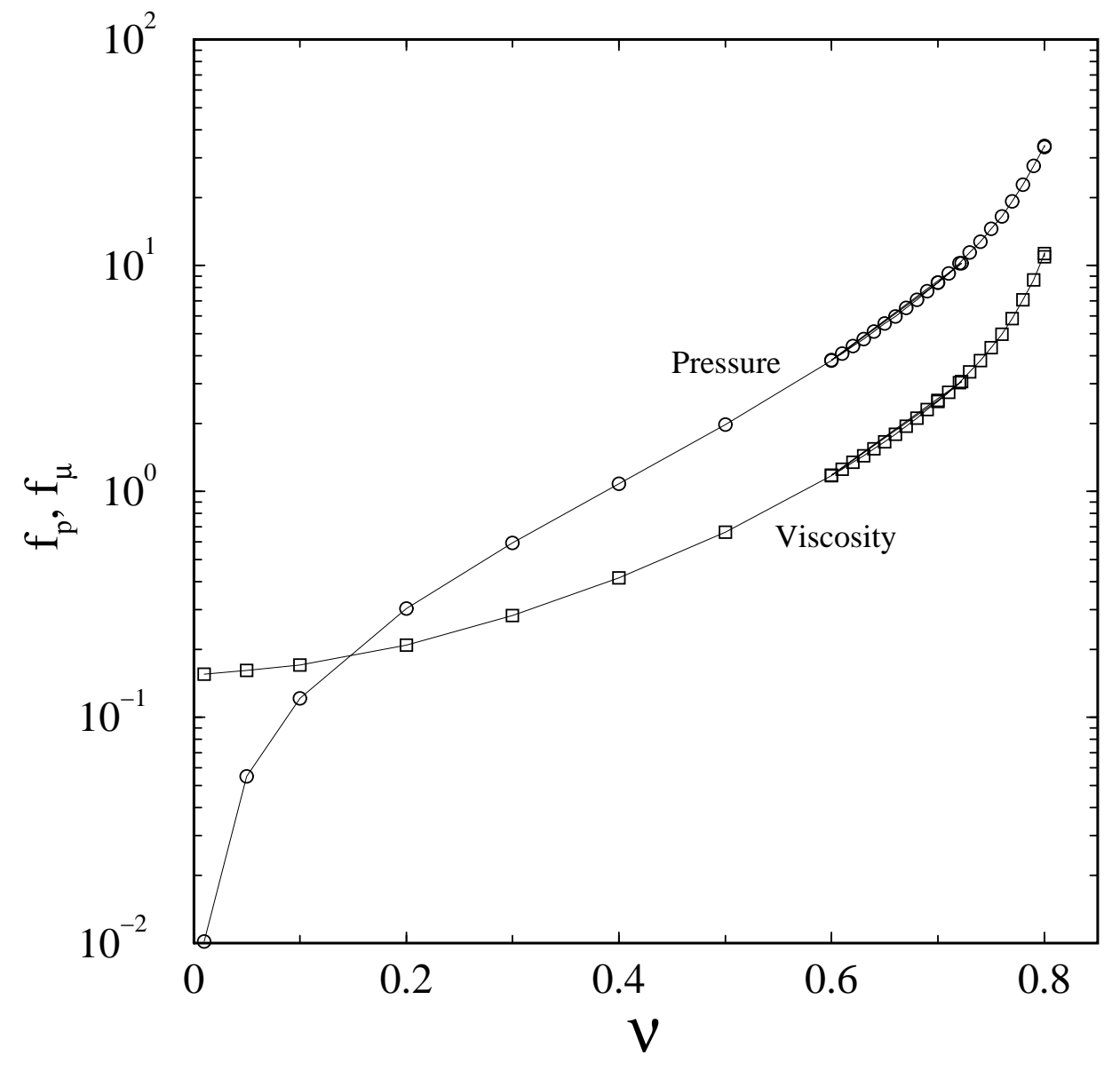

FIG. 6: Variations of the pressure and viscosity functions with density at $e=0.7: f_{p}=p / \rho T$ and $f_{\mu}=\mu / \rho \sigma \sqrt{T}$. The lines are drawn to guide the eye. 


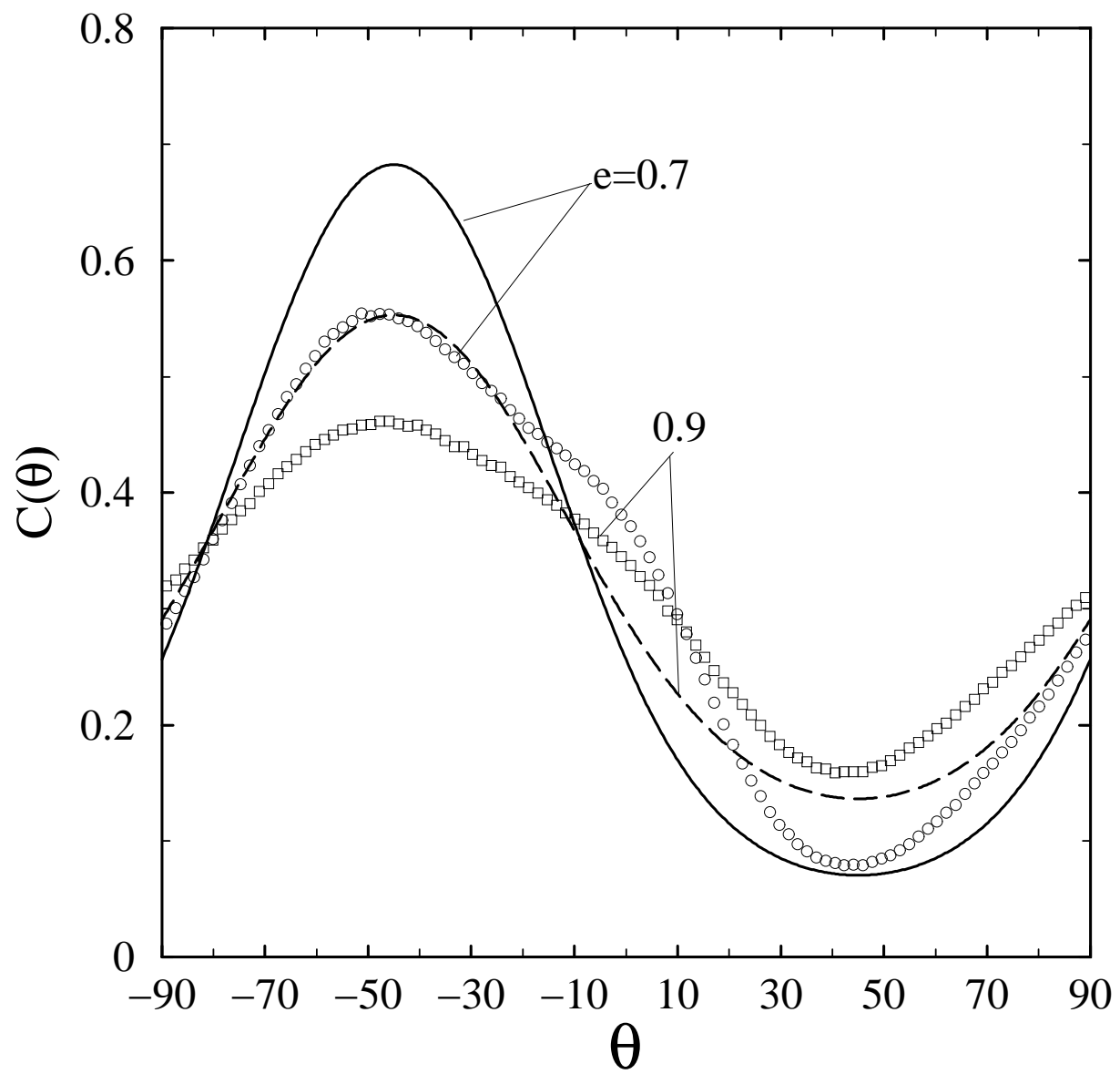

FIG. 7: Distribution of collision angles $C(\theta)$ for different coefficient of restitutions at $\nu=0.6$. The symbols represent simulation data and the lines theoretical predictions. 

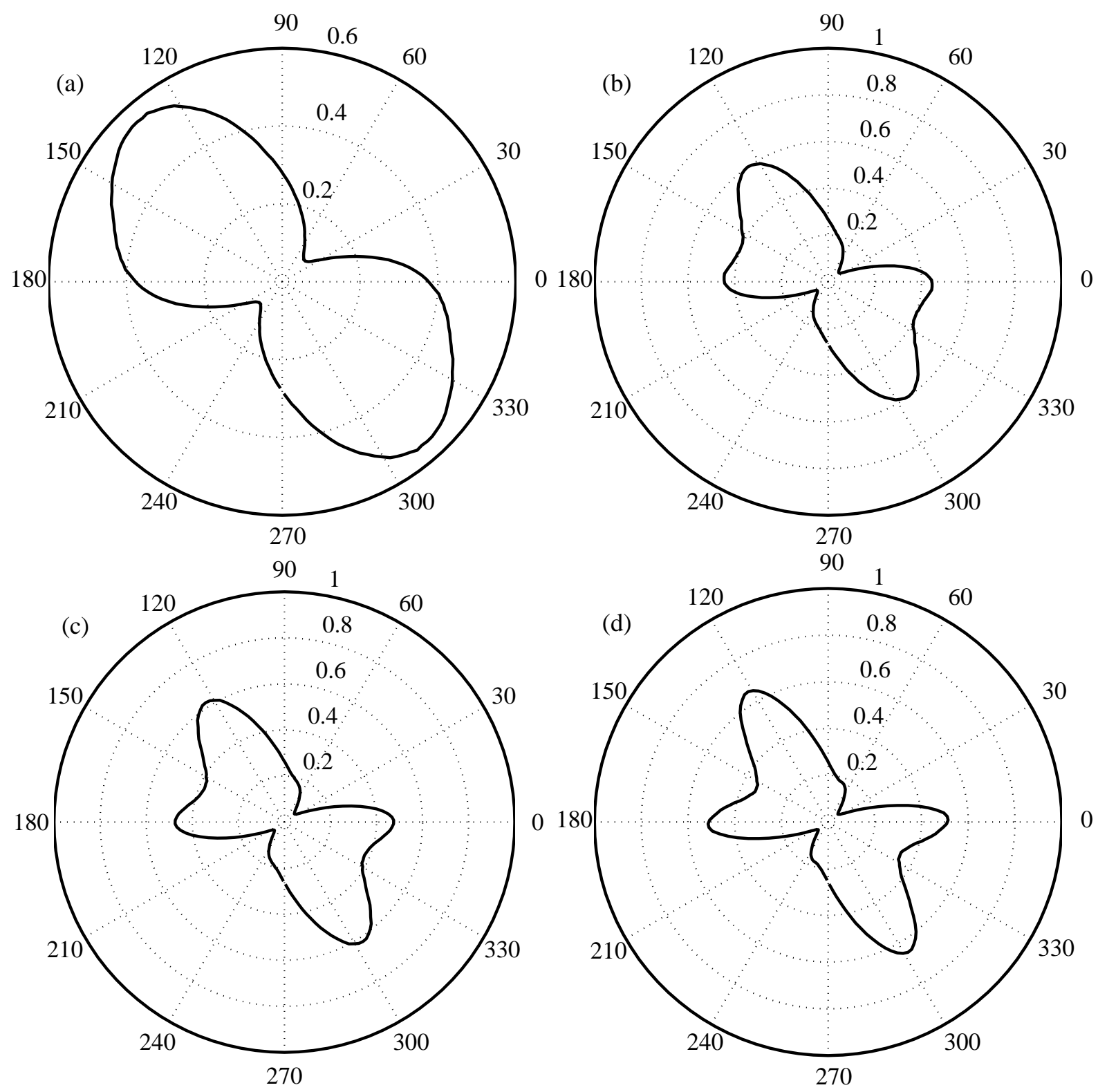

FIG. 8: Polar plots of the collision angle distribution for different densities at $e=0.7:(a) \nu=0.6$, (b) $\nu=0.7$; (c) $\nu=0.725$; (d) $\nu=0.75$. 


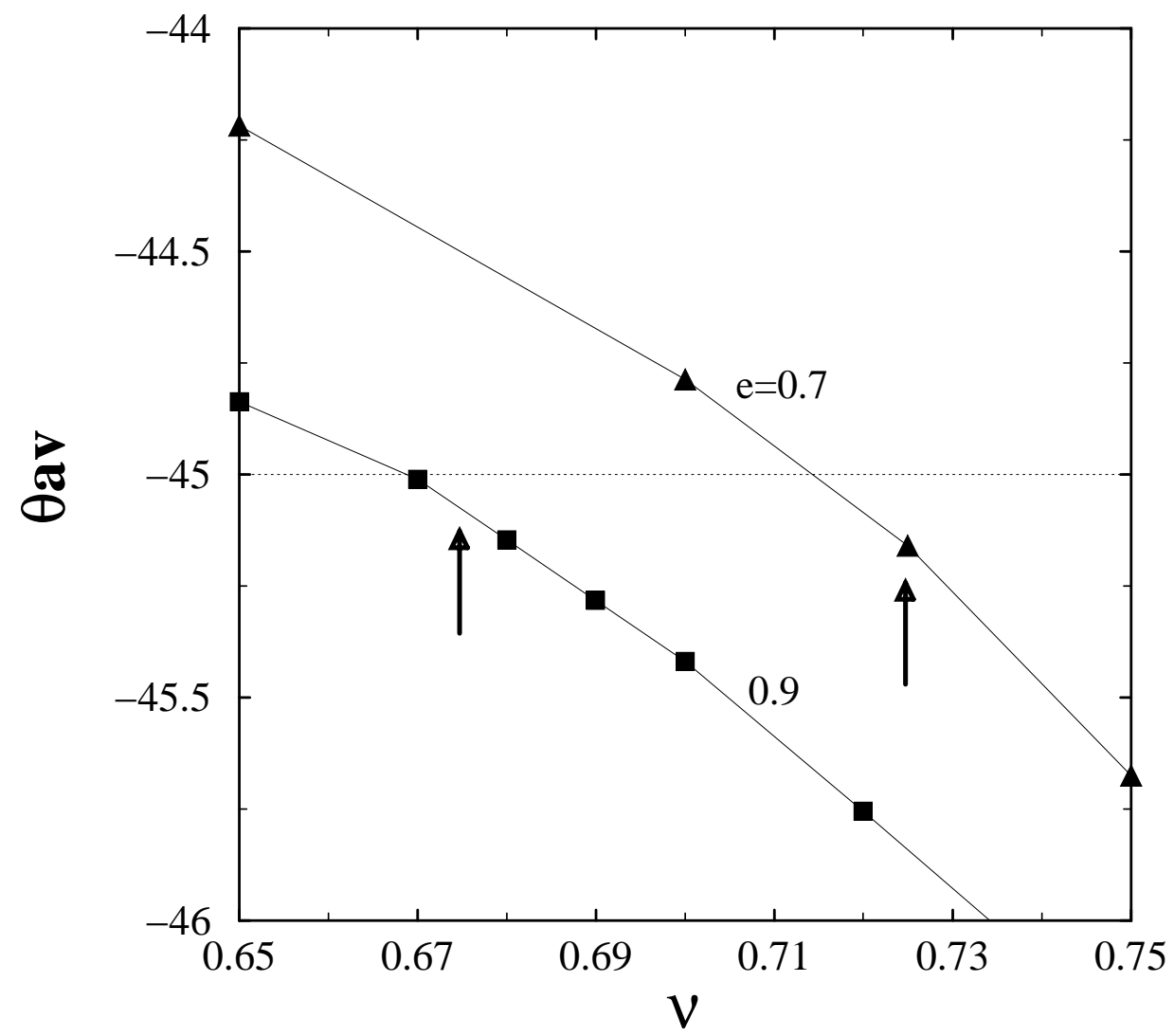

FIG. 9: Variations of the average collision angle $\theta_{a v}$ with density for different restitution coefficients. The arrows indicate densities where $\mathcal{N}_{1} \approx 0$. The lines joining the data points are to guide the eye. 

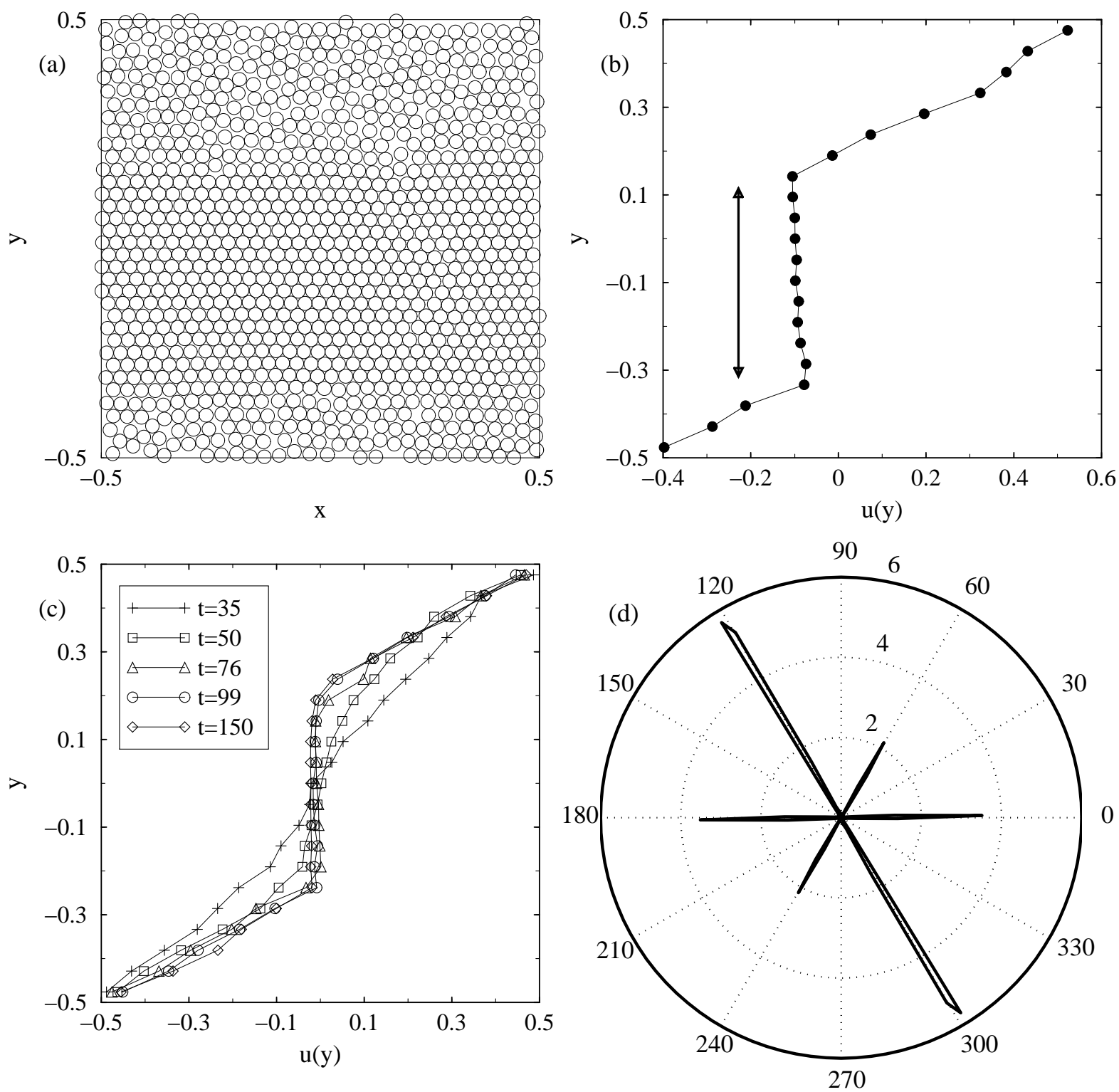

FIG. 10: Evidence of crystallization in a sheared dense granular fluid at $\nu=0.8$ and $e=0.9$. (a) Particle distribution and (b) streamwise velocity at $t=390$; (c) evolution of streamwise velocity at early times; (d) collision angle distribution. 

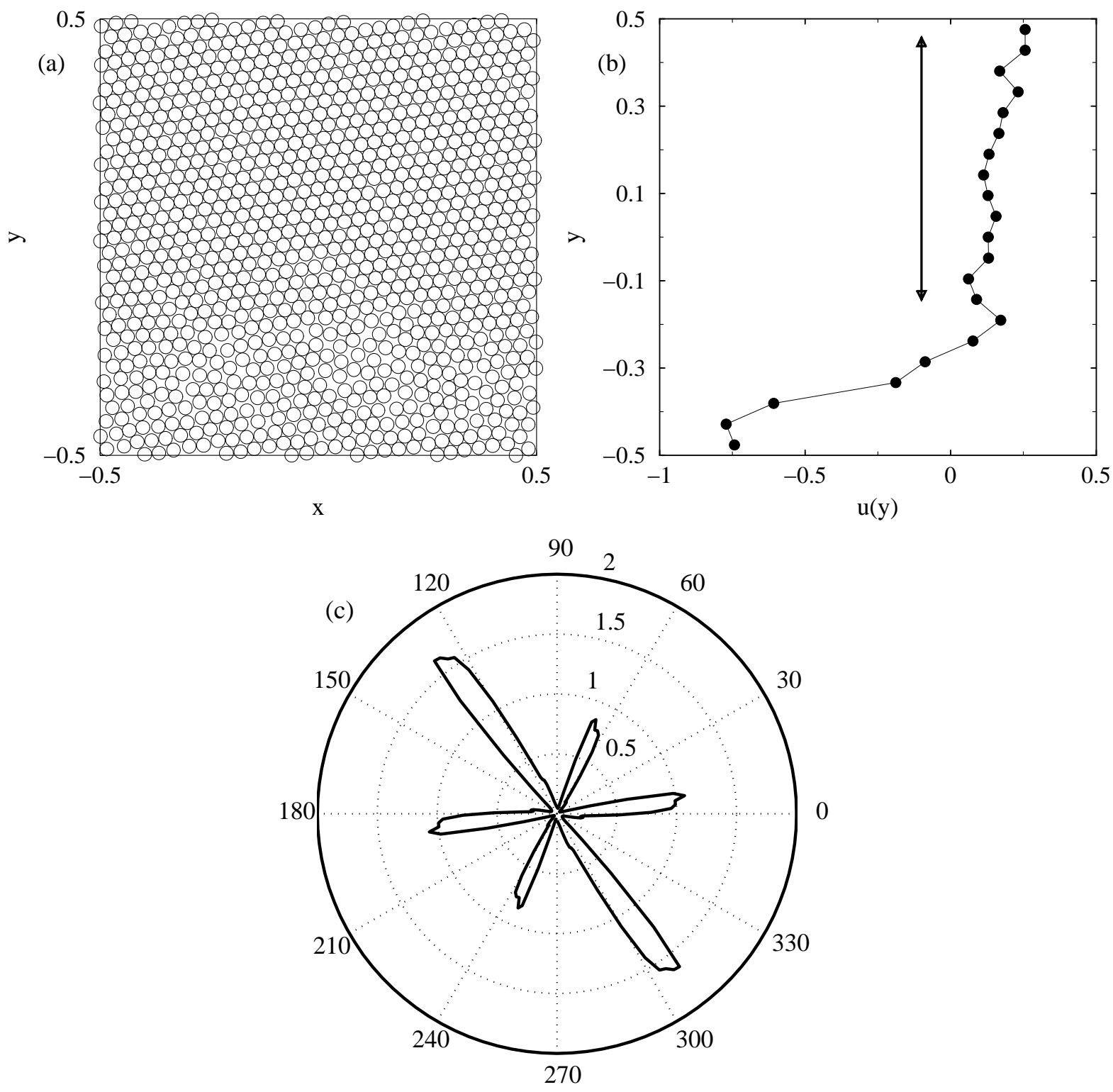

FIG. 11: Effect of the coefficient of restitution on crystallization: $\nu=0.8$ and $e=0.99$. (a) Particle distribution and (b) streamwise velocity at $t=150$; (c) collision angle distribution. 


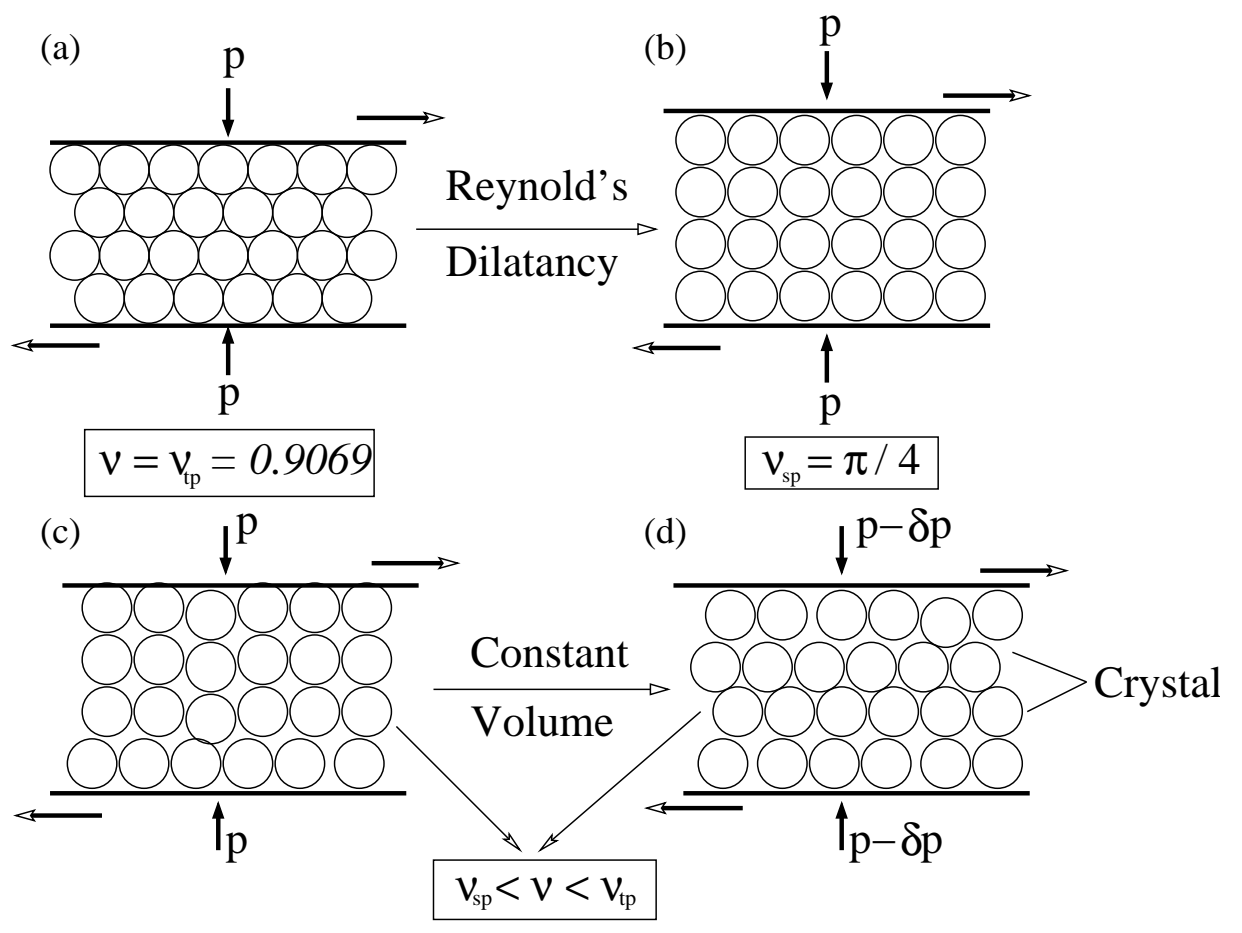

FIG. 12: A schematic diagram to explain Reynold's dilatancy in the Couette shear flow.

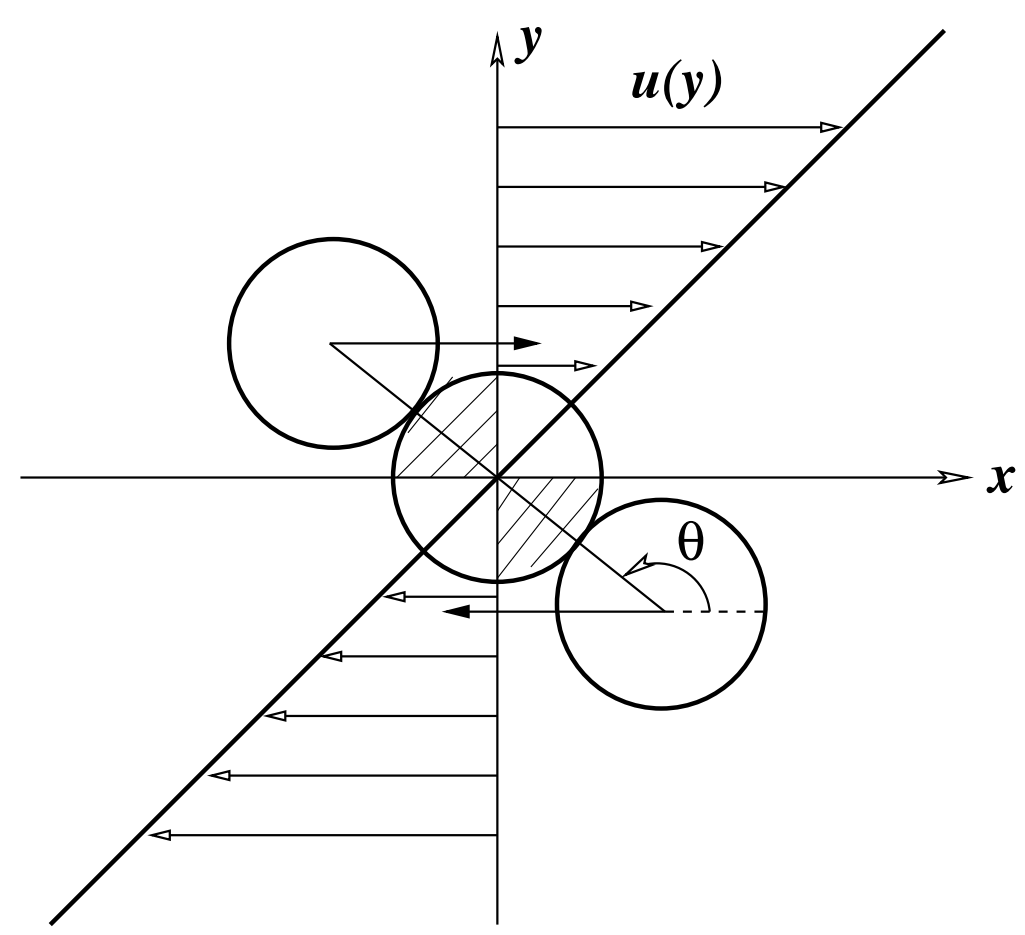

FIG. 13: A schematic of the possible collision angles $\theta$ in uniform shear flow; $\theta$ is measured anticlockwise from the positive $x$-axis. Note that the collisions are more likely to occur in the second $(\pi / 2<\theta<\pi)$ and fourth $(3 \pi / 2<\theta<2 \pi)$ quadrants of the colliding disks. 